Supplementary material for 'Planting the seed for sound change: Evidence from real-time MRI of velum kinematics in German', by Christopher Carignan, Stefano Coretta, Jens Frahm, Jonathan Harrington, Phil Hoole, Arun Joseph, Esther Kunay, and Dirk Voit. Language 97(2).333-64, 2021.

\title{
Supplement for Planting the seed for sound change: evidence from real-time MRI of velum kinematics in German
}

\section{Contents}

1 Prepare data $\quad 2$

2 Velum gesture duration $\quad 2$

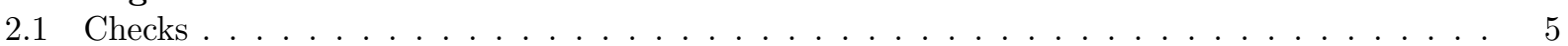

3 Velum gesture onset $\quad 6$

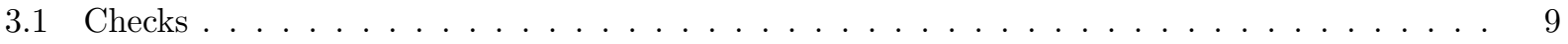

4 Velum gesture peak (timing) 10

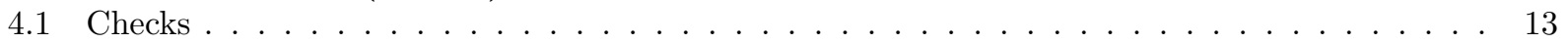

5 Velum gesture offset $\quad 14$

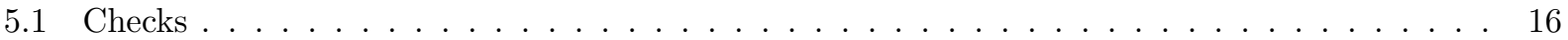

6 Velum gesture peak (magnitude) 18

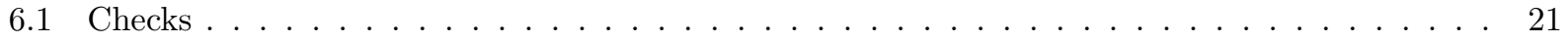

7 Gesture offset stiffness $\quad 22$

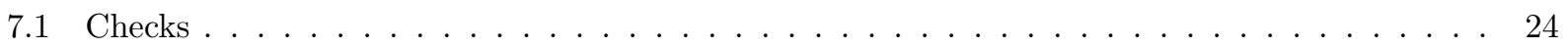

8 Gesture offset stiffness $\quad 26$

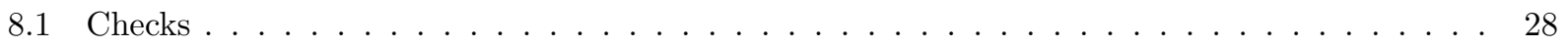

9 Kurtosis $\quad 30$

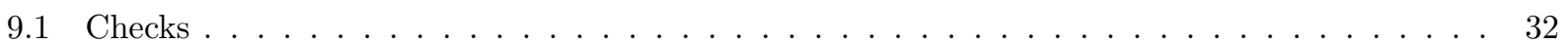

10 Crest factor $\quad 34$

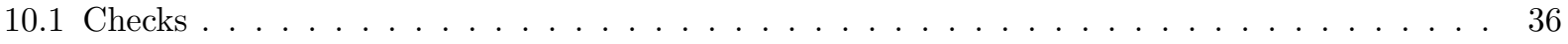

11 Integral of velum movement in vowel (area under the curve) 38

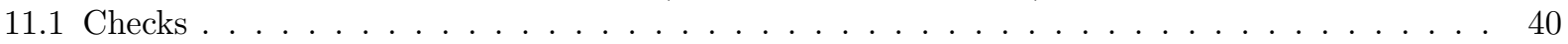

12 Plotting posteriors $\quad 42$

12.1 Figure 5: duration, onset, peak (timing), offset $\ldots \ldots \ldots \ldots \ldots \ldots \ldots$

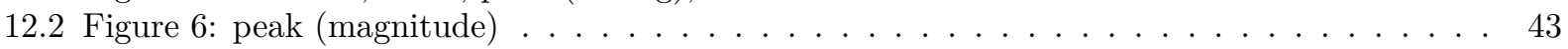

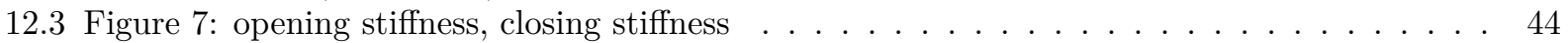

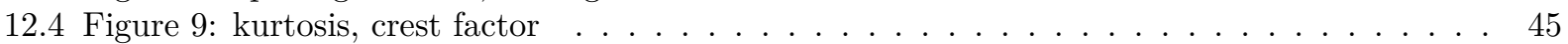

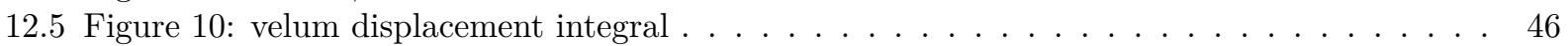

References $\quad 46$

knitr: :opts_chunk\$set (echo = TRUE)

knitr: :opts_knit\$set(root.dir = here: :here())

library (tidyverse)

library (patchwork) 


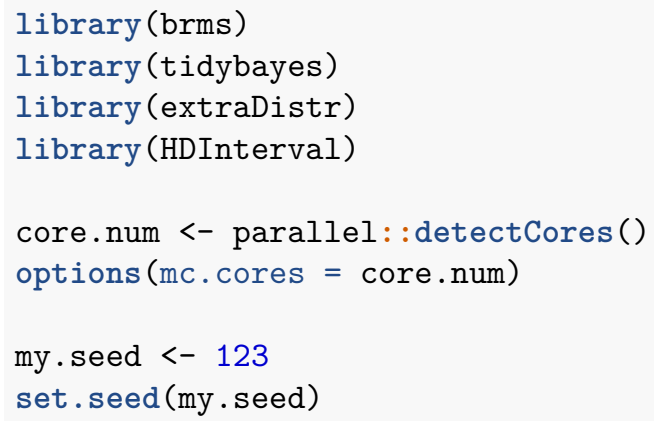

\section{Prepare data}

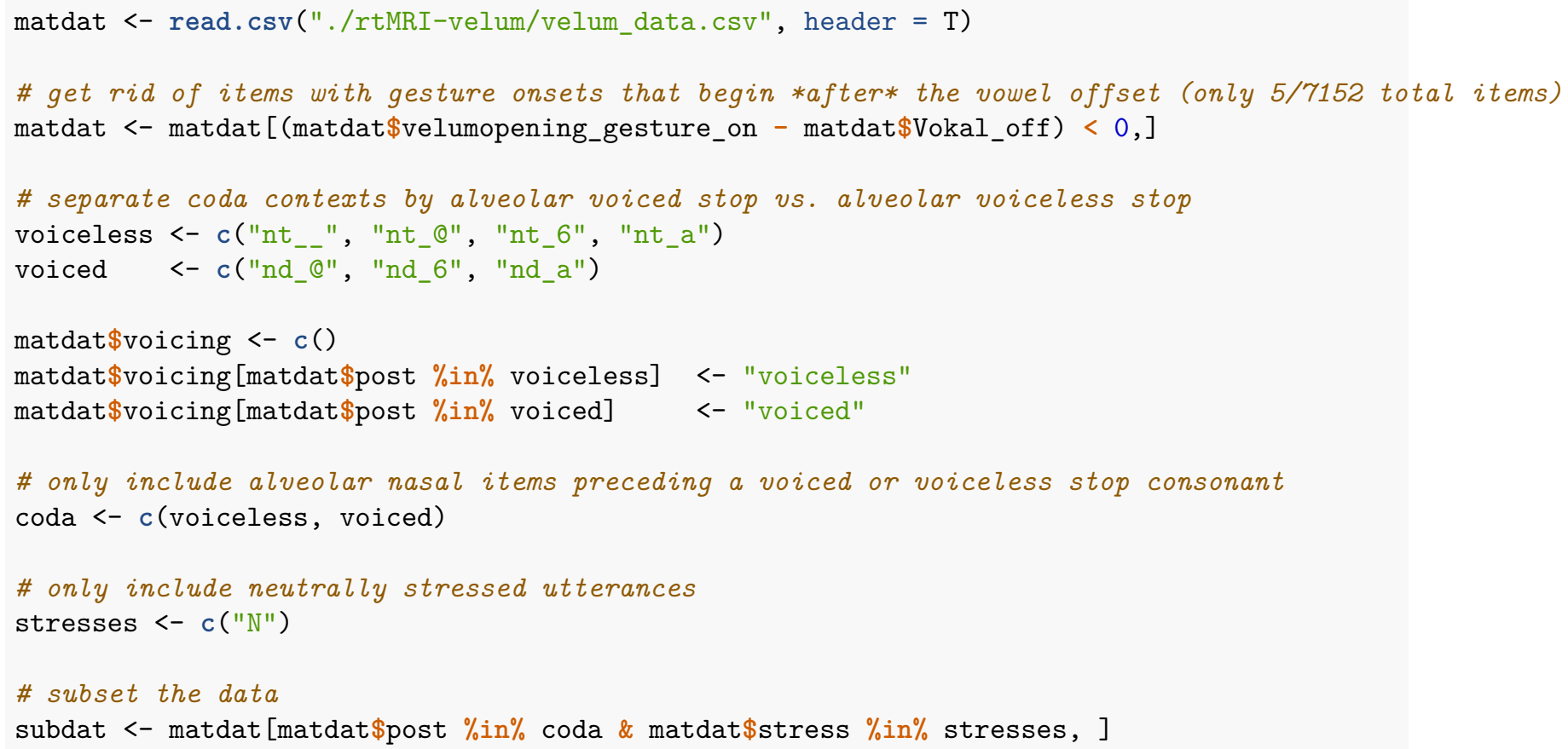

\section{Velum gesture duration}

Since no specific information on expectations for velum gesture duration can be found in the literature, we followed more general expectations for speech segment durations, coded as very weakly informative priors. The BRM for velum gesture duration was built using a log-normal distribution, since speech segment duration has been shown to be log-normally distributed (Rosen 2005; Ratnikova 2017; Gahl and Baayen 2019). The following distributions were used as weakly informative priors (on the log-odds scale): for the intercept of duration (corresponding to ND), a normal distribution with mean $=0$ and standard deviation $=3(\operatorname{Normal}(0,3))$; for the effect of voicing (when NT), $\operatorname{Normal}(0,1)$. These roughly correspond to a belief that the intercept of velum gesture duration (when the context is ND) is between 0 and $400 \mathrm{~ms}\left(e^{3 \times 2}=403\right)$, and the duration changes (increases or decreases) by a factor of 1 to 7.4 in the ND context $\left(e^{1 \times 2}=7.4\right)$, at $95 \%$ confidence. For the model standard deviation and the random intercept standard deviation we used a half-Cauchy distribution with location 0 and scale 0.1 (HalfCauchy $(0,0.1)$ ); this corresponds to a $95 \%$ HDI $=[0,2.55]$ in log-odds, i.e. a factor change of 1 to $12.75\left(e^{2} .55=12.75\right)$. 
inverseCDF (c(0.025, 0.975), phcauchy, sigma $=0.1)$

\#\# [1] $0.003930135 \quad 2.545175934$

For the correlation between random effects, we used an $L K J(2)$ distribution, as recommended by Vasishth et al. (2018). The same prior specification was used for the models of velum gesture onset time and velum gesture offset time.

NOTE: Warnings about $\hat{R}$ and sample size are false warnings, and are not signs of problems with fitting and they can safely be ignored. See https://github.com/paul-buerkner/brms/issues/864.

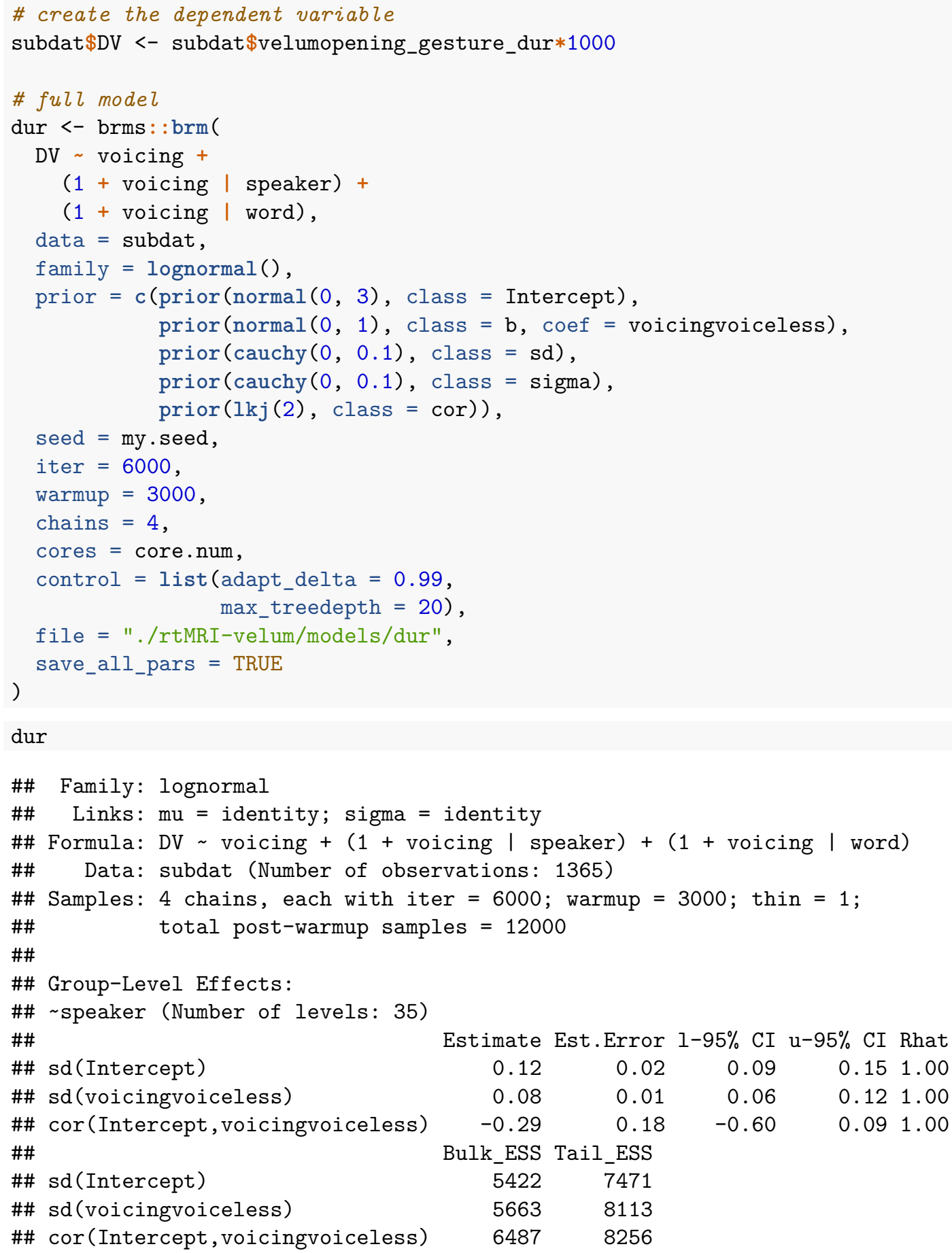




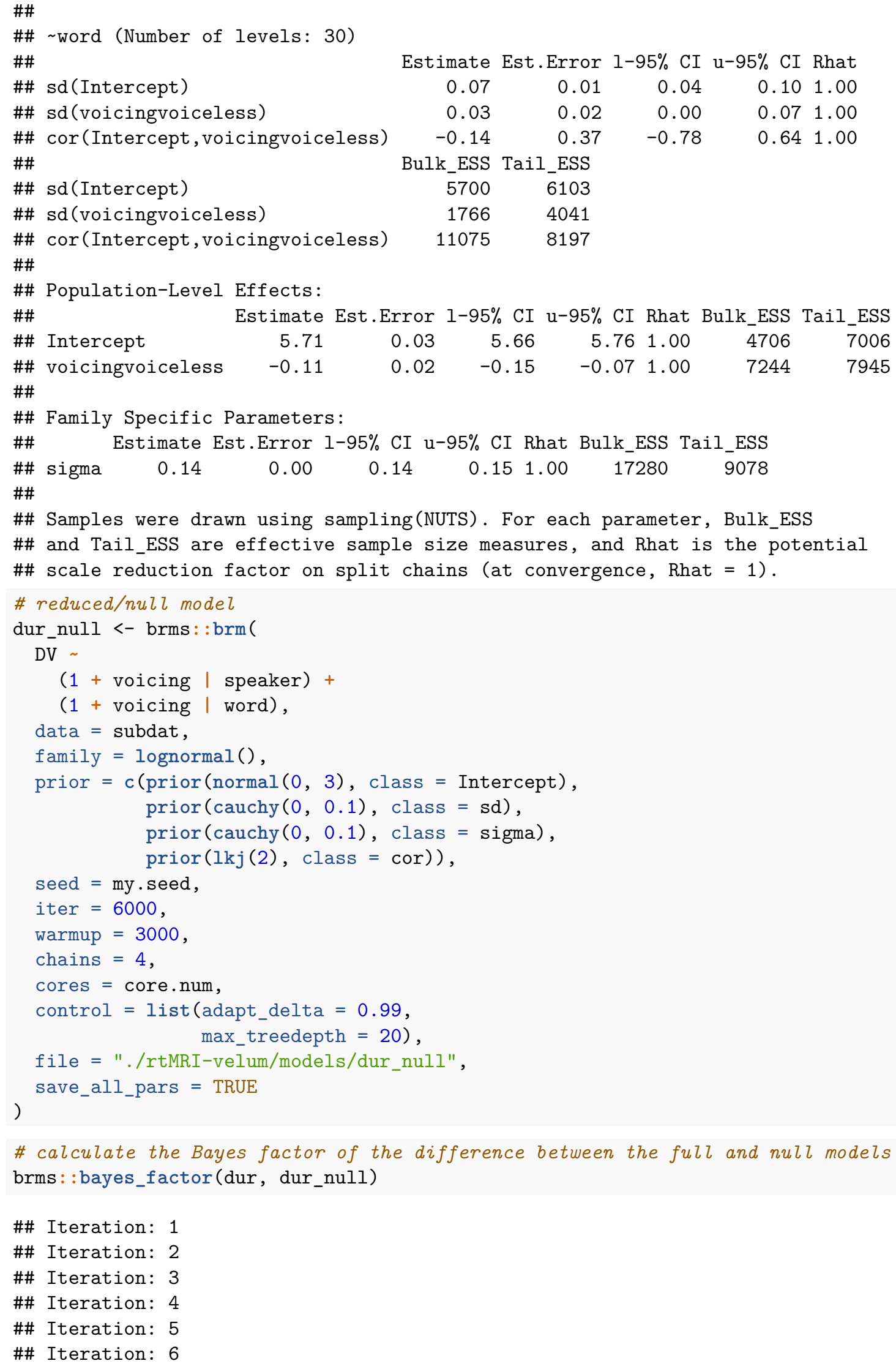




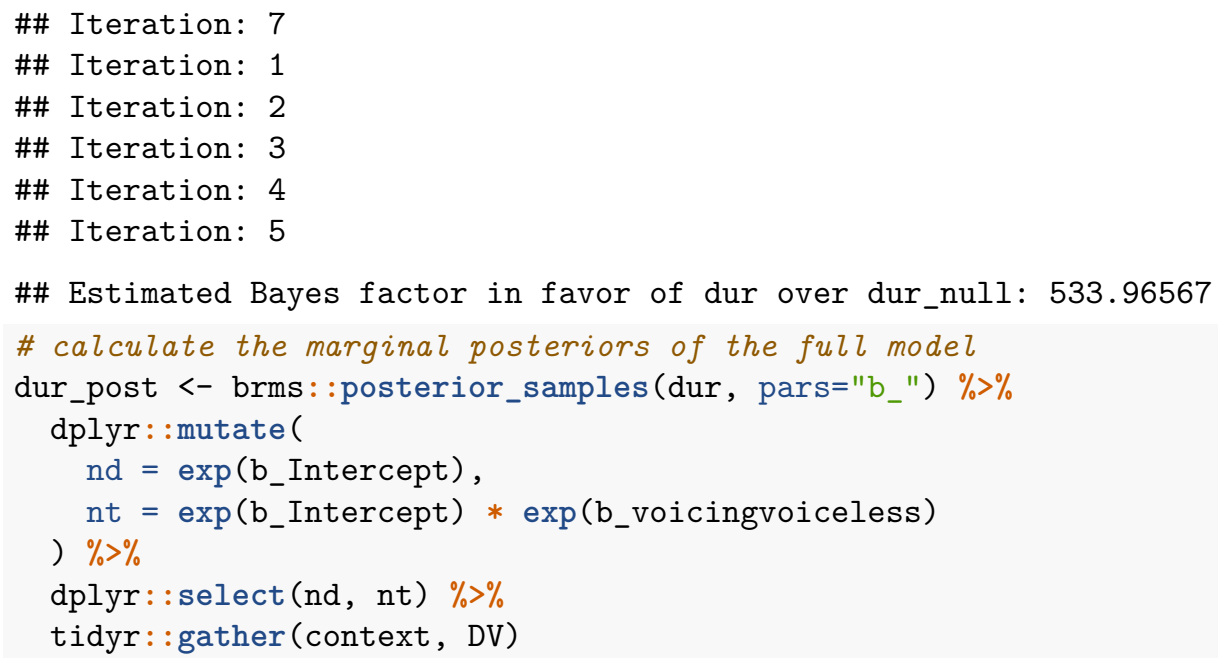

\subsection{Checks}

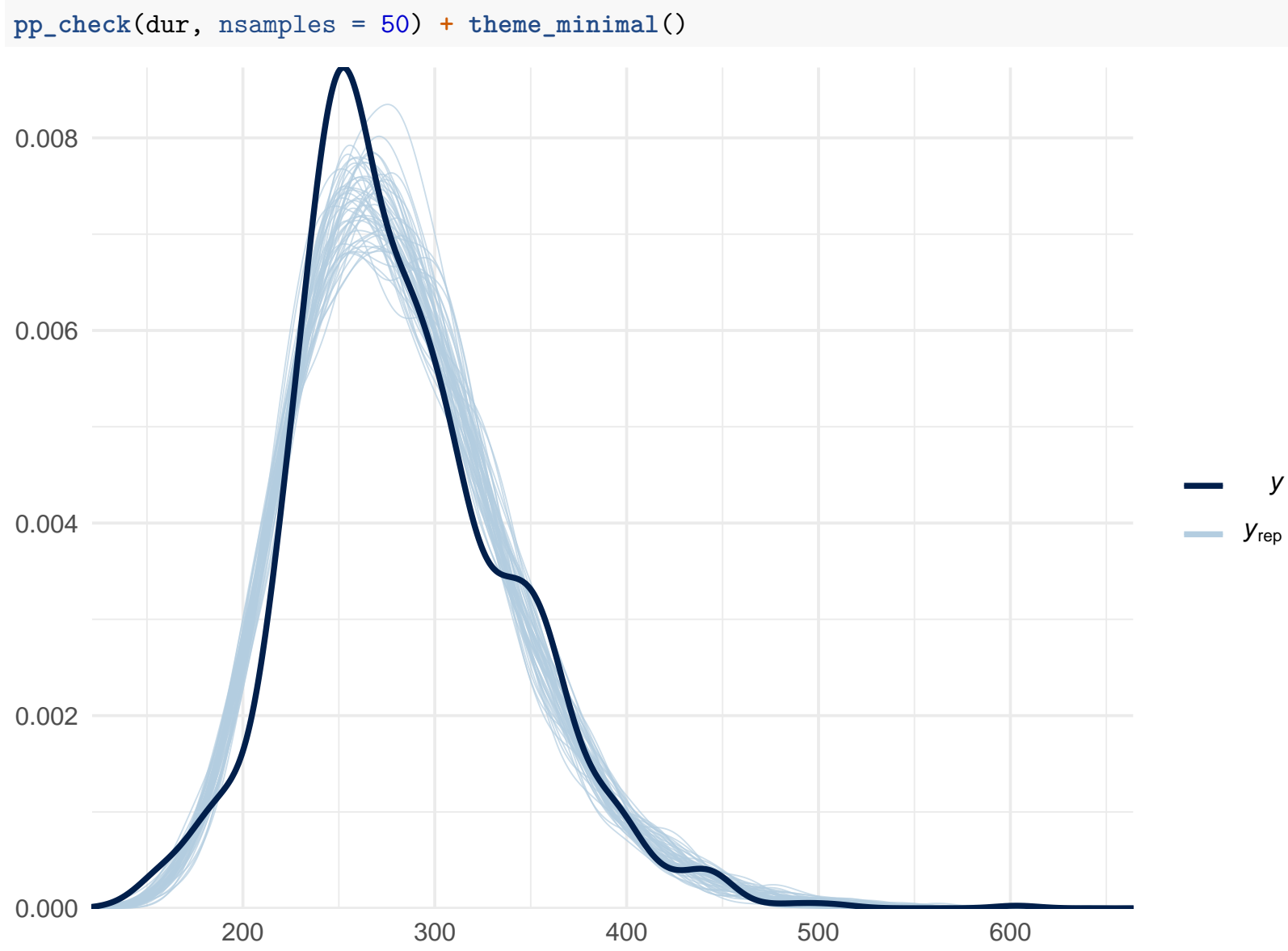

For the sensitivity analysis we followed the method introduced in Betancourt (2018). In the following plot, $s$ (posterior shrinkage) indicates the contribution of the data to the posterior (values closer to 1 are better). $z$ (posterior z-score) indicates how close the inference is to the data generating process (lower values are better). An ideal fit should have $s=1$ and $z=0$. Posteriors with high $s$ and high $z$ indicate overfit (the data dominate the posterior), while posteriors with low $s$ and low $z$ indicate that the model is poorly 
identified (the data is not contributing to the model and the priors dominate).

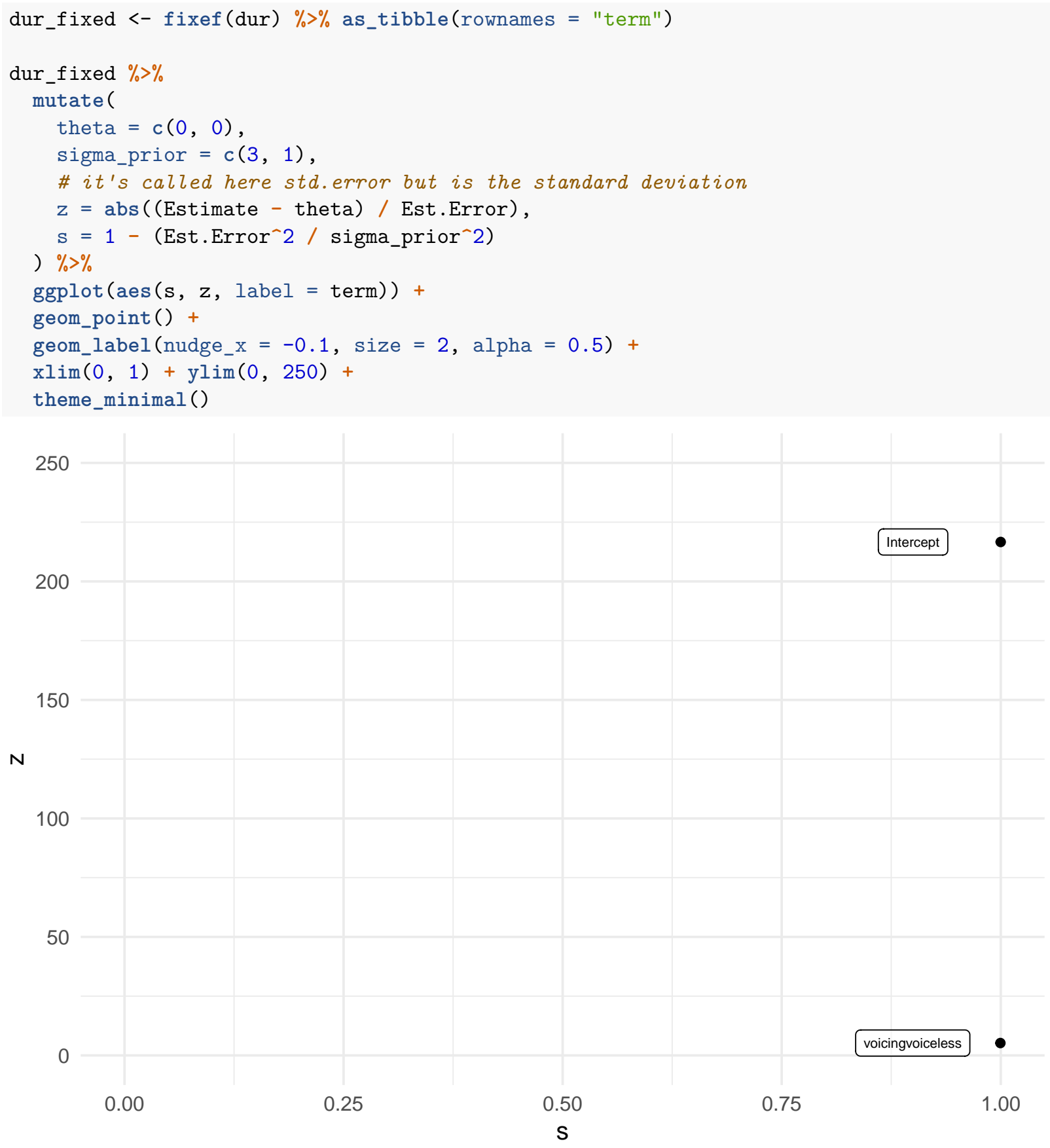

\section{Velum gesture onset}

See velum gesture duration for prior specification.

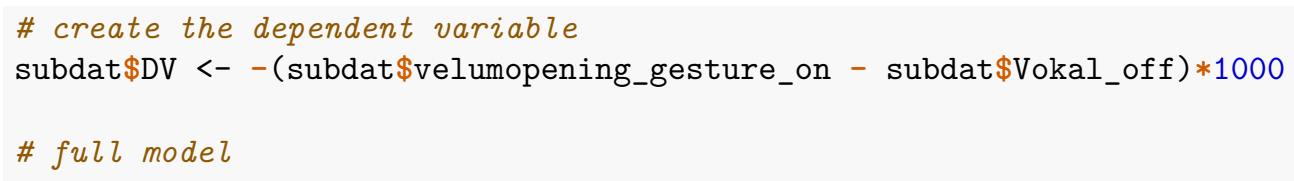




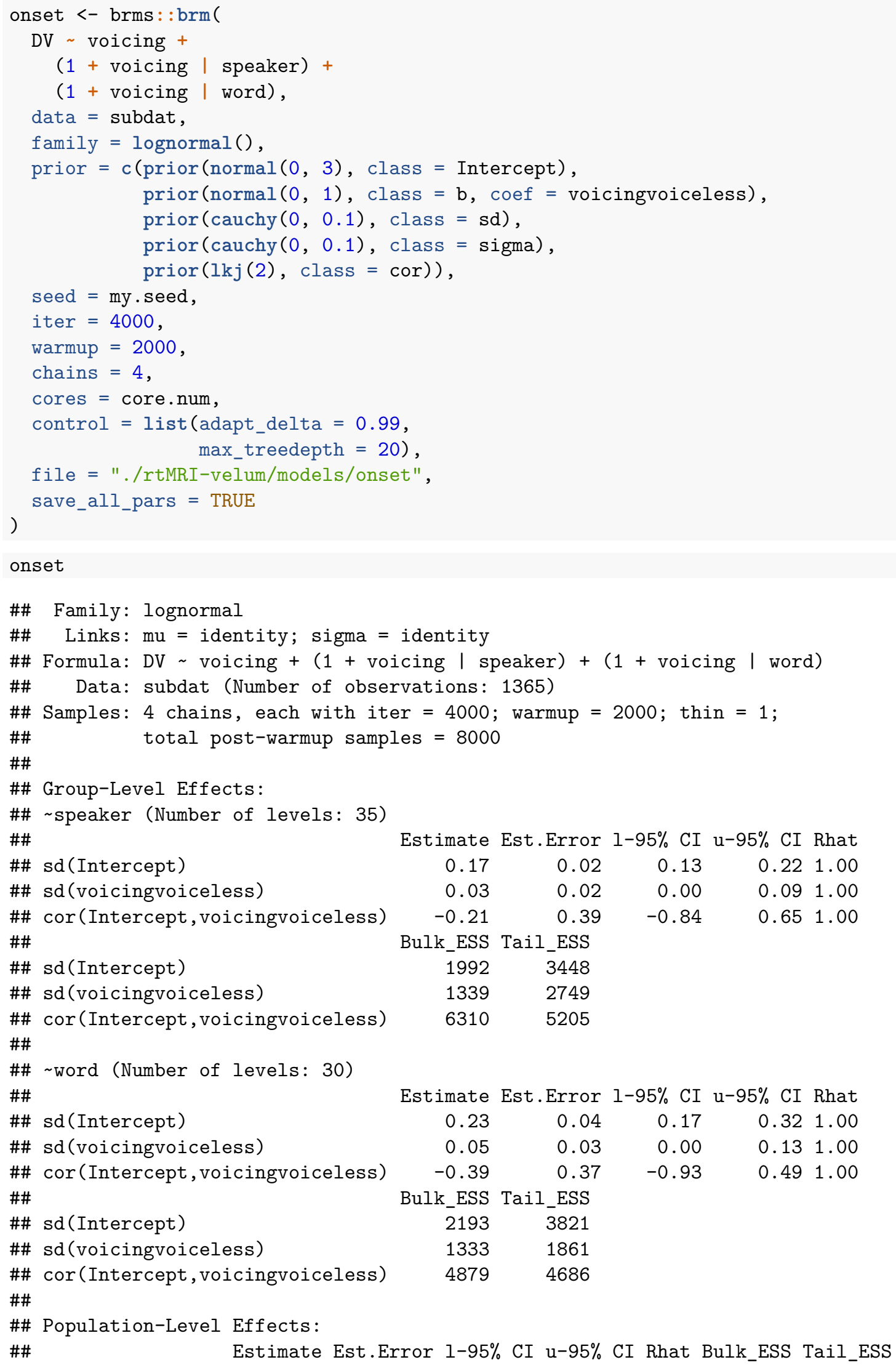




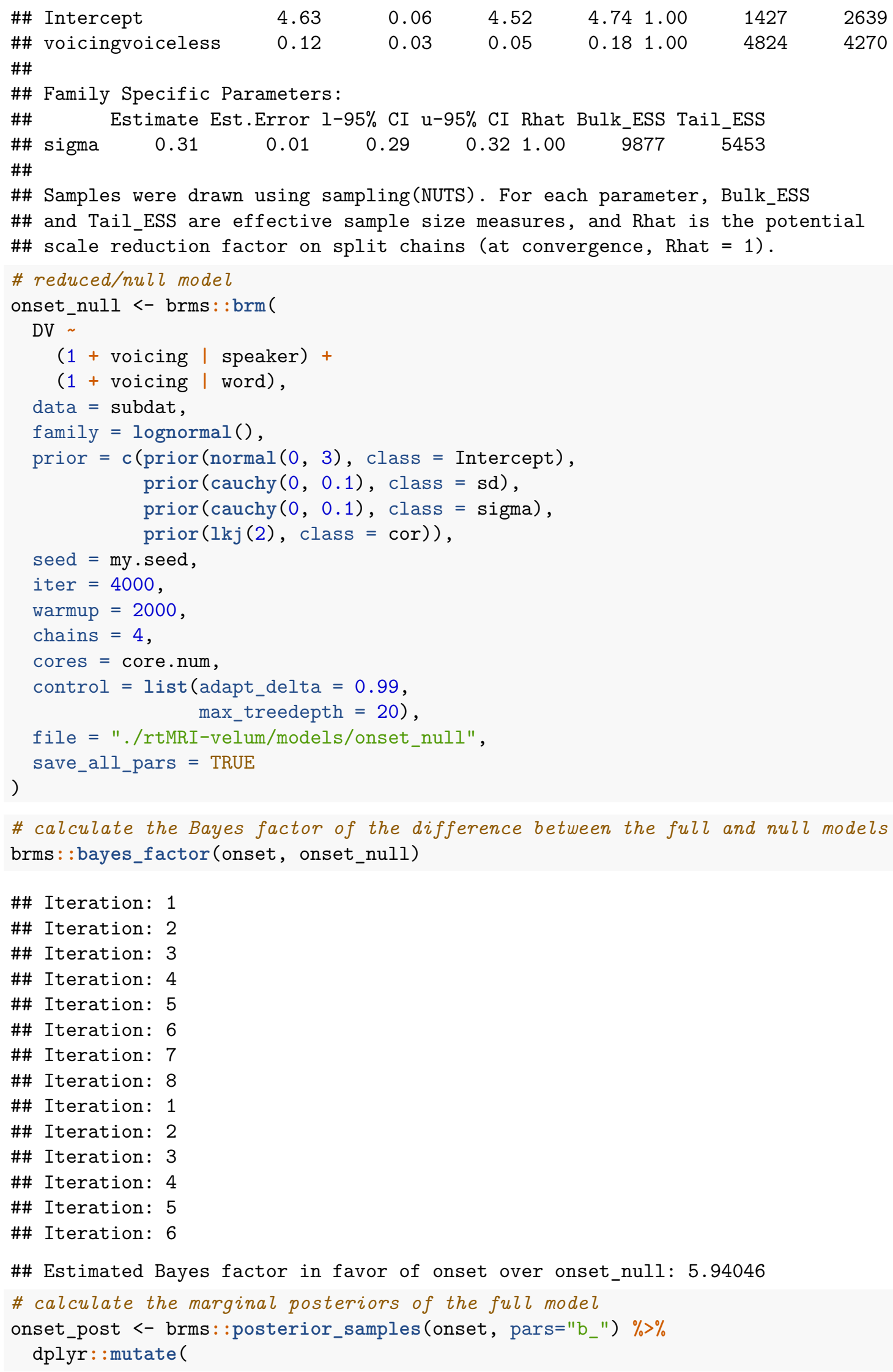




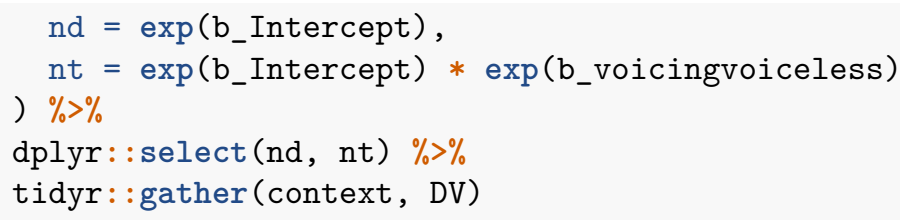

\subsection{Checks}

pp_check (onset, nsamples $=50)+$ theme_minimal $($ )

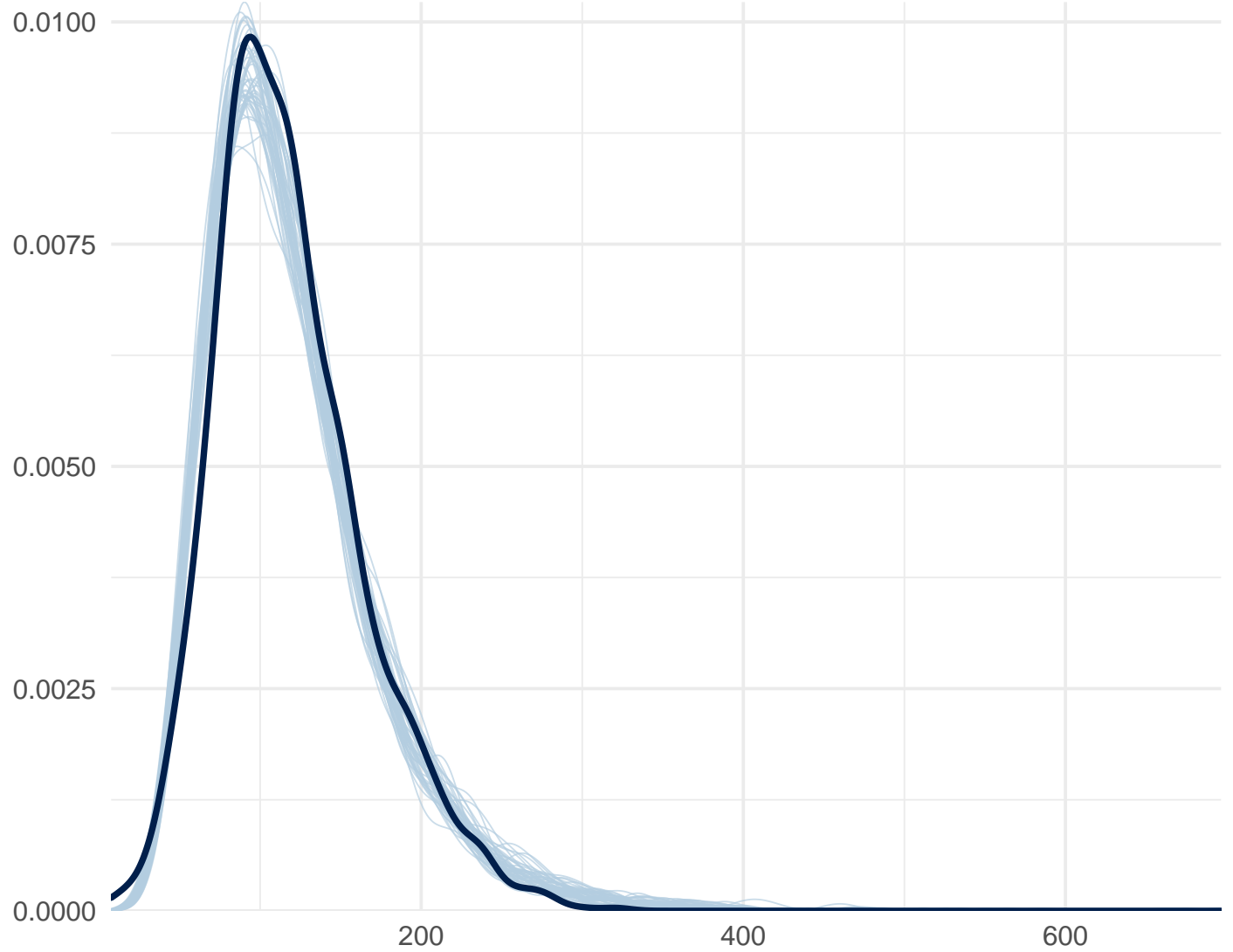

onset_fixed <- fixef(onset) $\%>\%$ as_tibble (rownames = "term")

onset_fixed $\%>\%$

mutate (

theta $=c(0,0)$,

sigma_prior $=c(3,1)$,

\# it's called here std.error but is the standard deviation

$\mathrm{z}=\operatorname{abs}($ (Estimate - theta) / Est.Error),

$s=1-$ (Est.Error`2 / sigma_prior`2)

) $\%>\%$

$\operatorname{ggplot}(\operatorname{aes}(\mathbf{s}, \mathbf{z}$, label $=$ term $))+$

geom_point() +

geom_label(nudge_x $=-0.1$, size $=2$, alpha $=0.5)+$

$\mathrm{x} \lim (0,1)+\mathrm{y} \lim (0,250)+$

theme_minimal () 


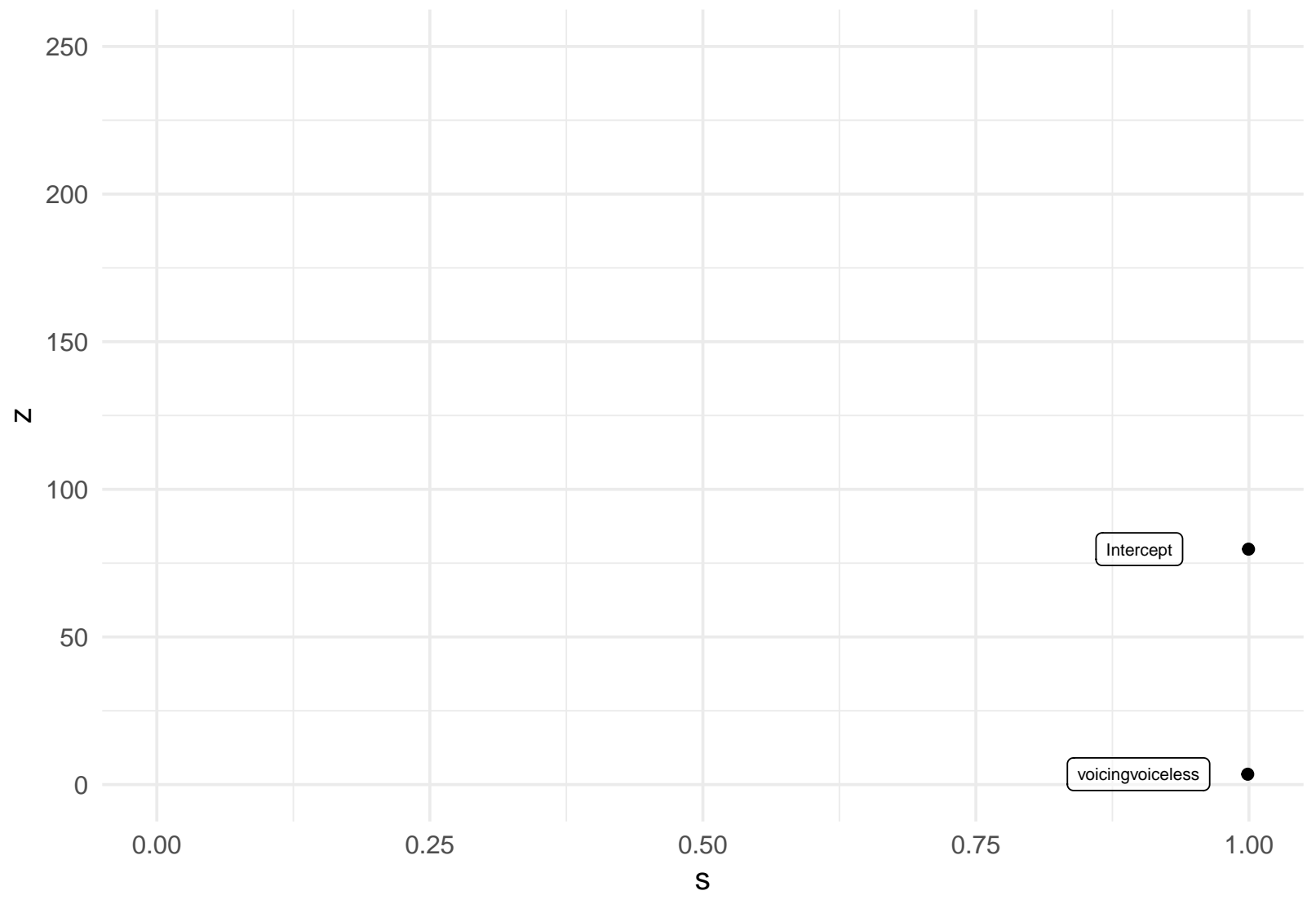

\section{Velum gesture peak (timing)}

The BRM for the time point of the velum gesture peak was built using a Gaussian distribution; unlike the other measures of timing, the time point of the velum gesture peak is not expected to follow a onesided (positive support) distribution, since the peak can potentially occur before or after the vowel offset. The following distributions were used as weakly informative priors (on the milliseconds scale): for the intercept (corresponding to ND), Normal $(0,200)$; for the effect of voicing (when NT), Normal $(0,100)$. These correspond to a belief that the intercept is between -400 and $400 \mathrm{~ms}$ from the vowel offset, and that the time changes by -200 to $200 \mathrm{~ms}$ in the NT context, at $95 \%$ confidence. For the model standard deviation and the random intercept standard deviation we used HalfCauchy $(0,5)$, which corresponds to a $95 \%$ HDI $=[0,127] \mathrm{ms}$.

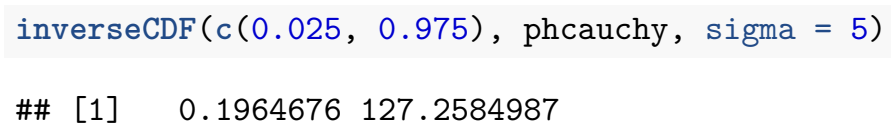

For the correlation between random effects, an $L K J(2)$ distribution.

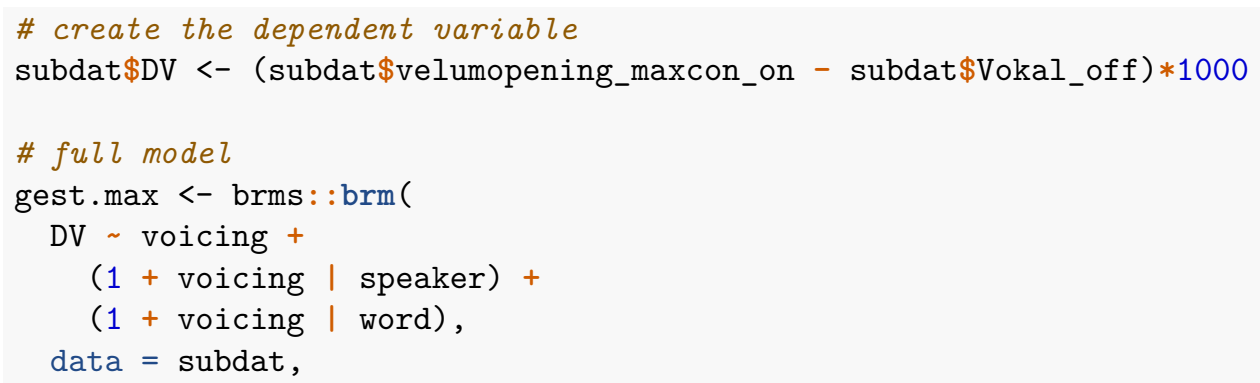




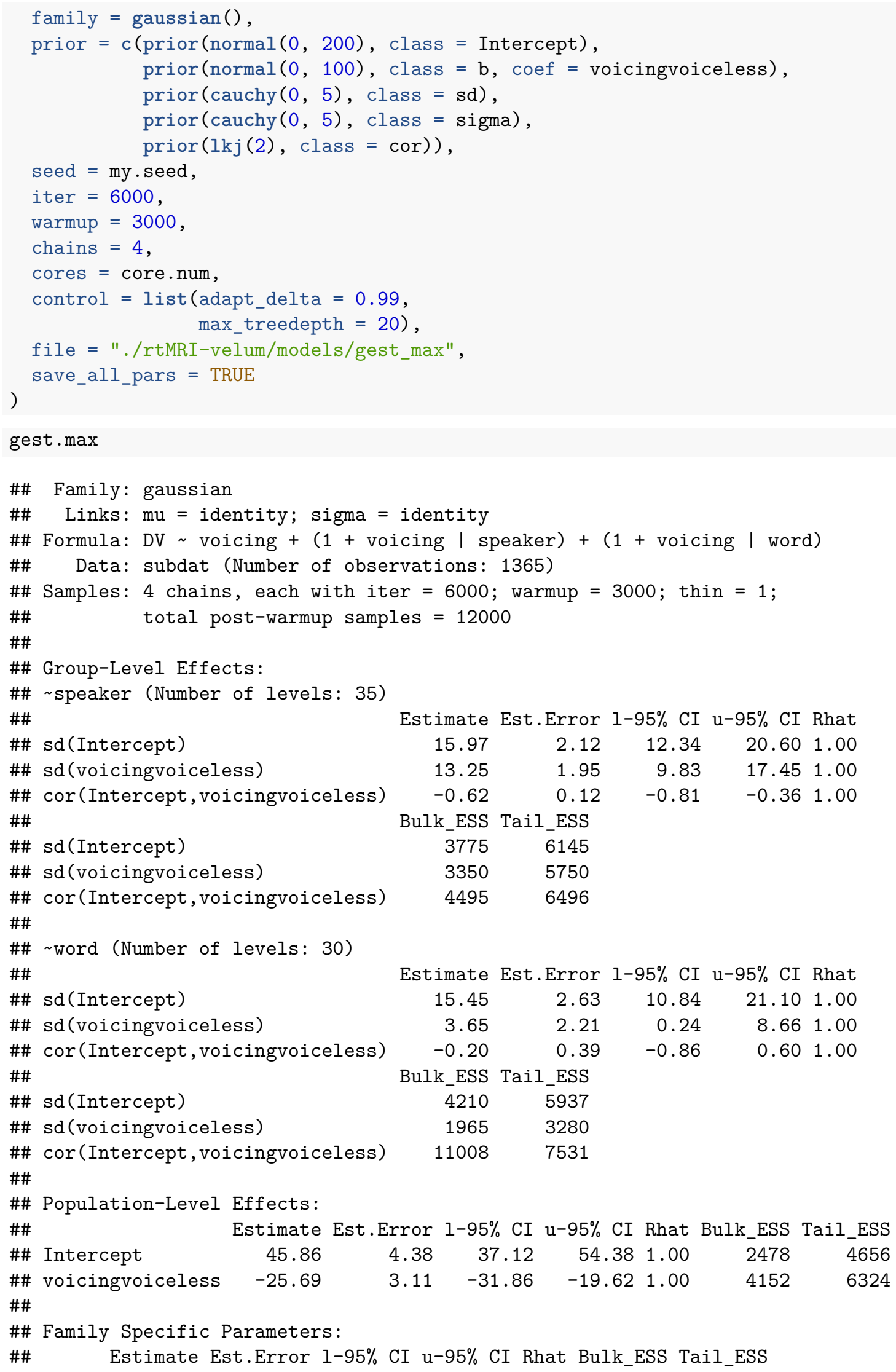




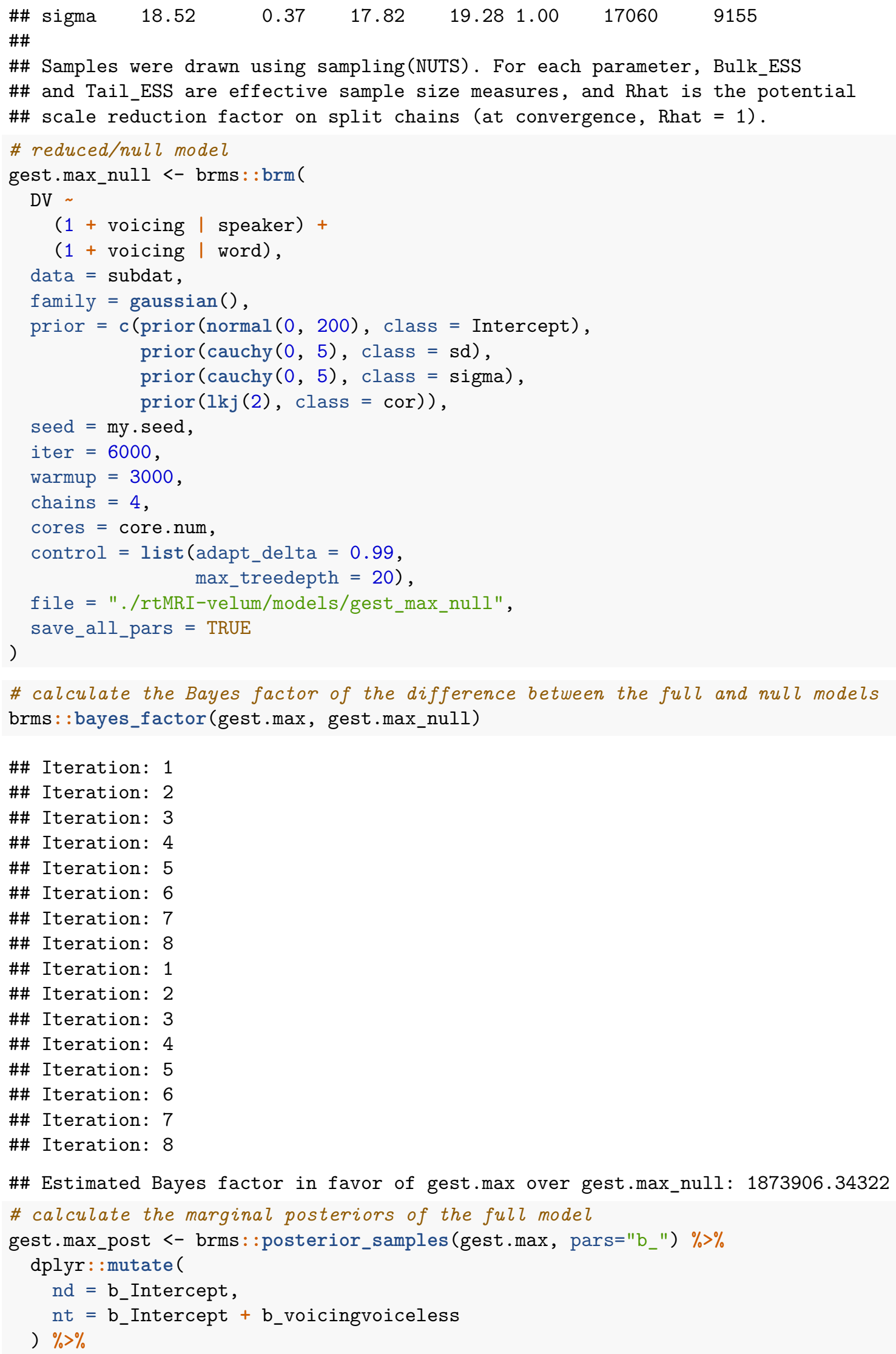


dplyr: : select (nd, nt) $\%>\%$

tidyr: :gather (context, DV)

\subsection{Checks}

pp_check(gest.max, nsamples $=50)+$ theme_minimal ()

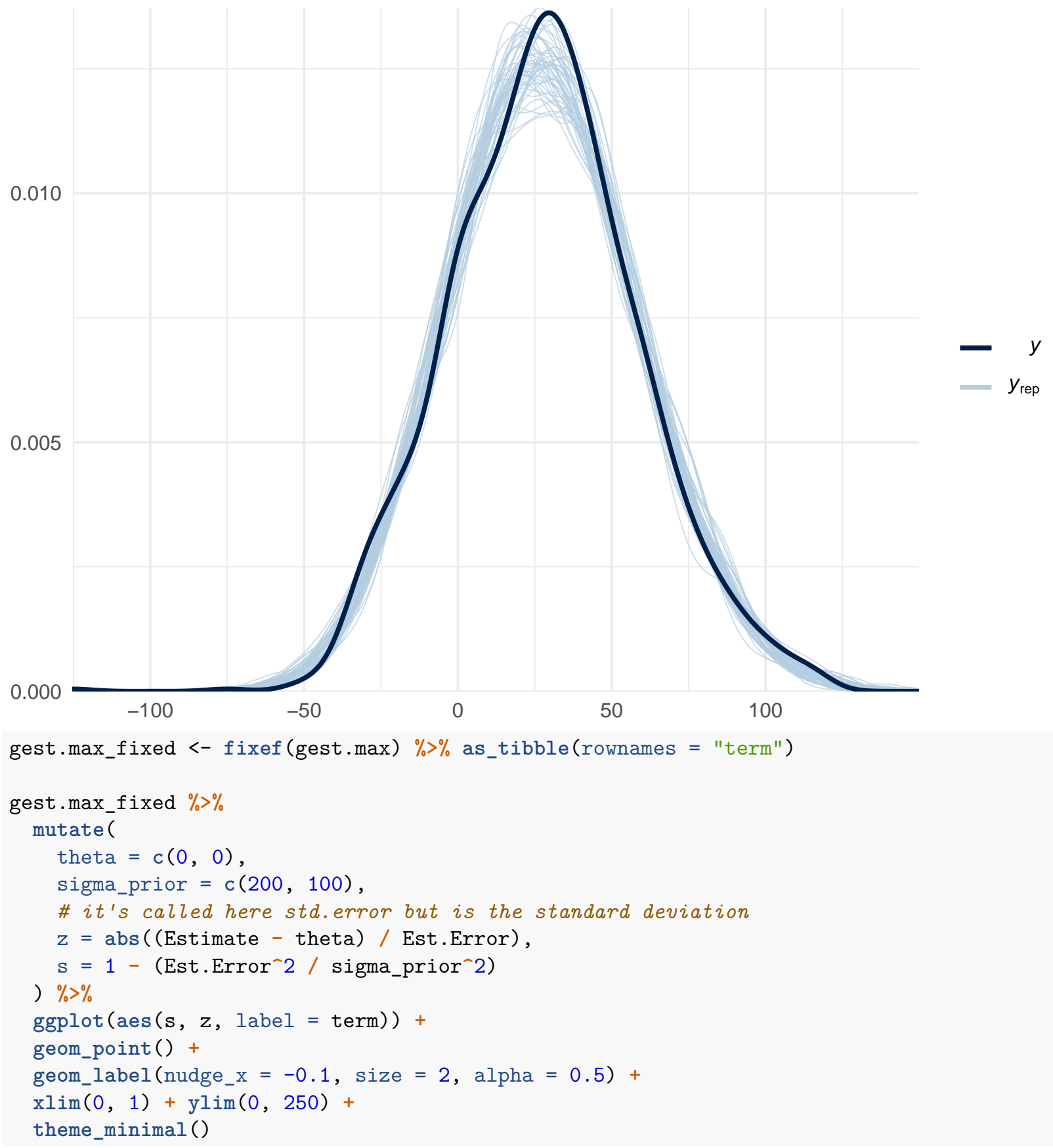




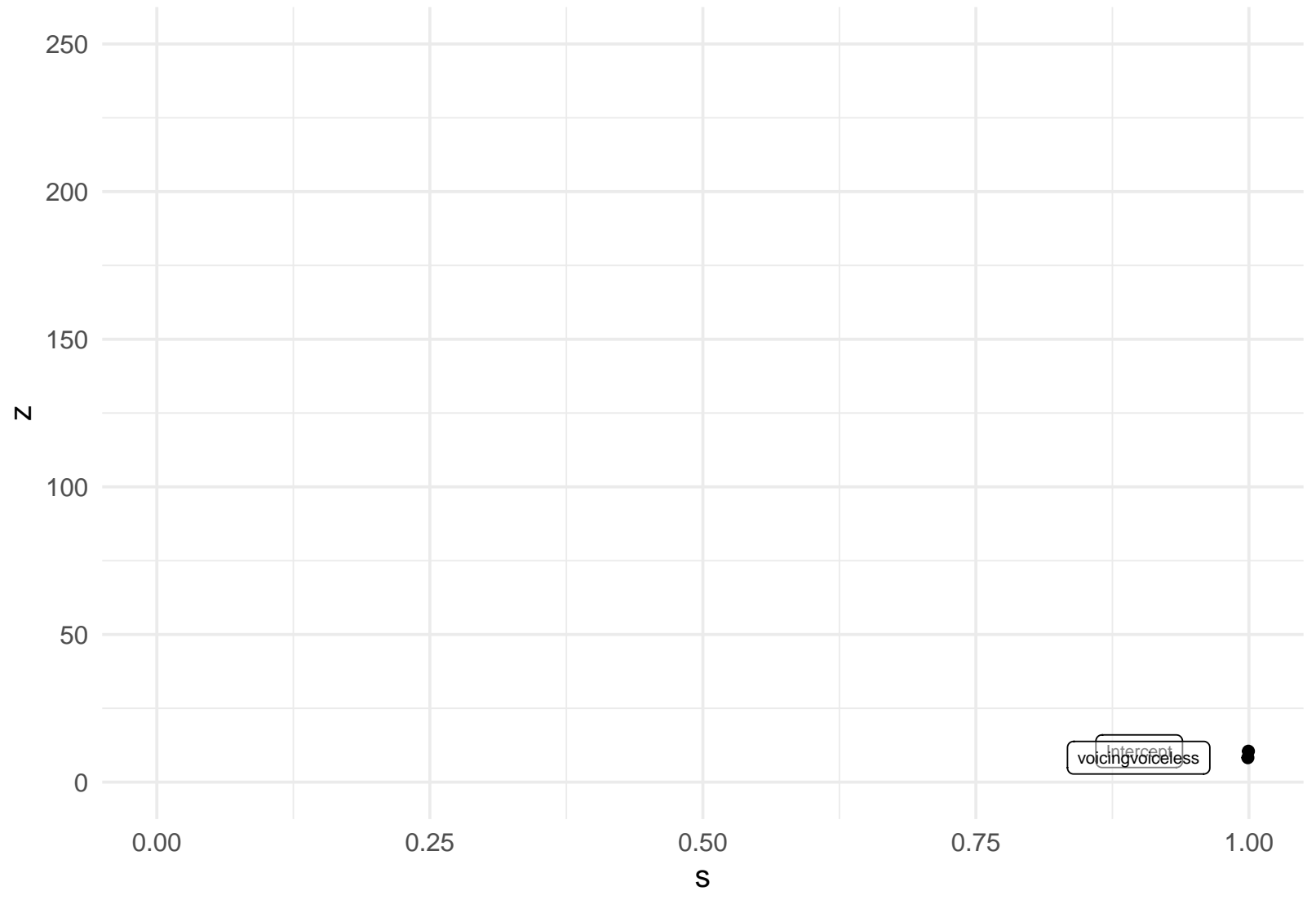

\section{$5 \quad$ Velum gesture offset}

See velum gesture duration for prior specification.

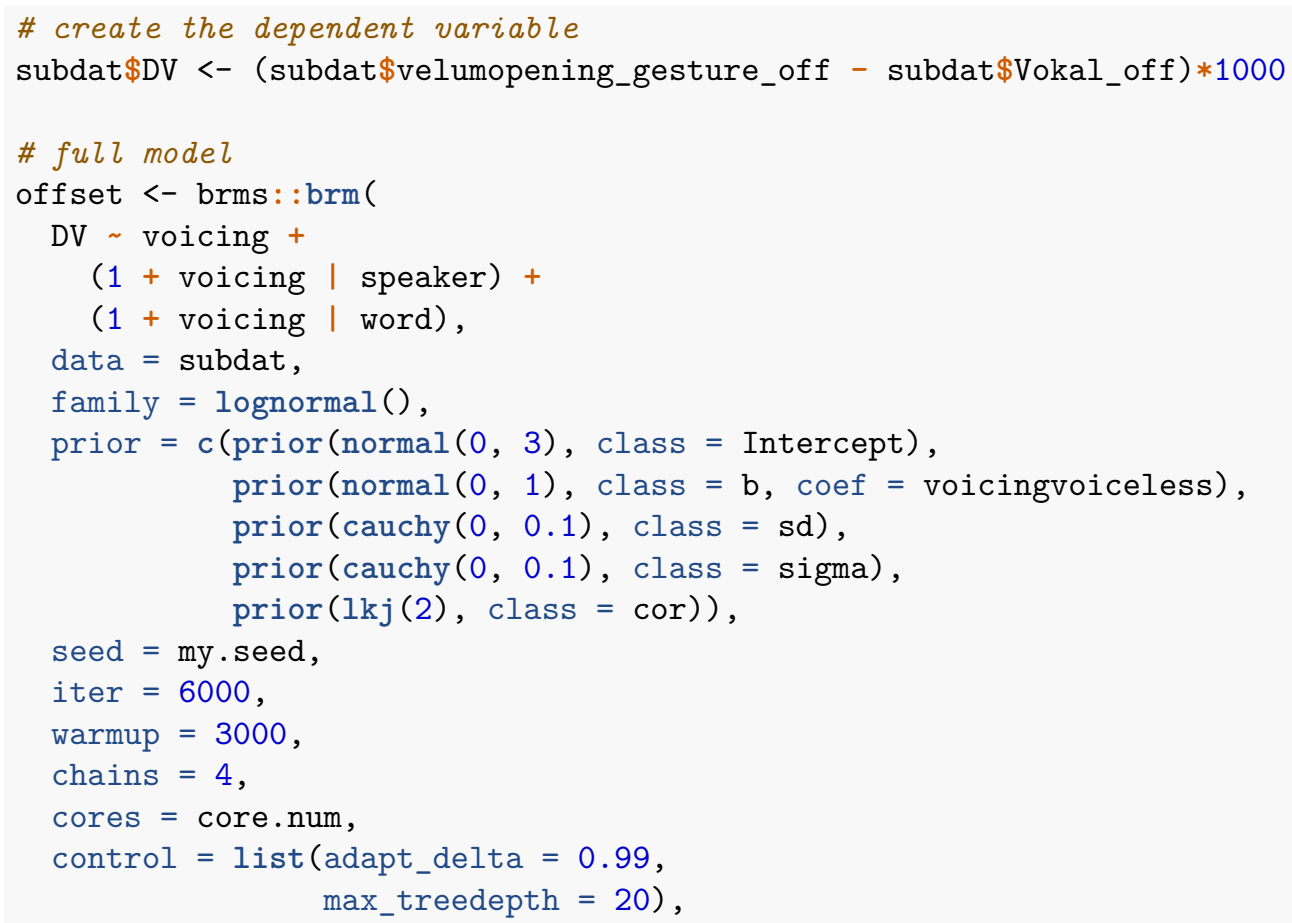




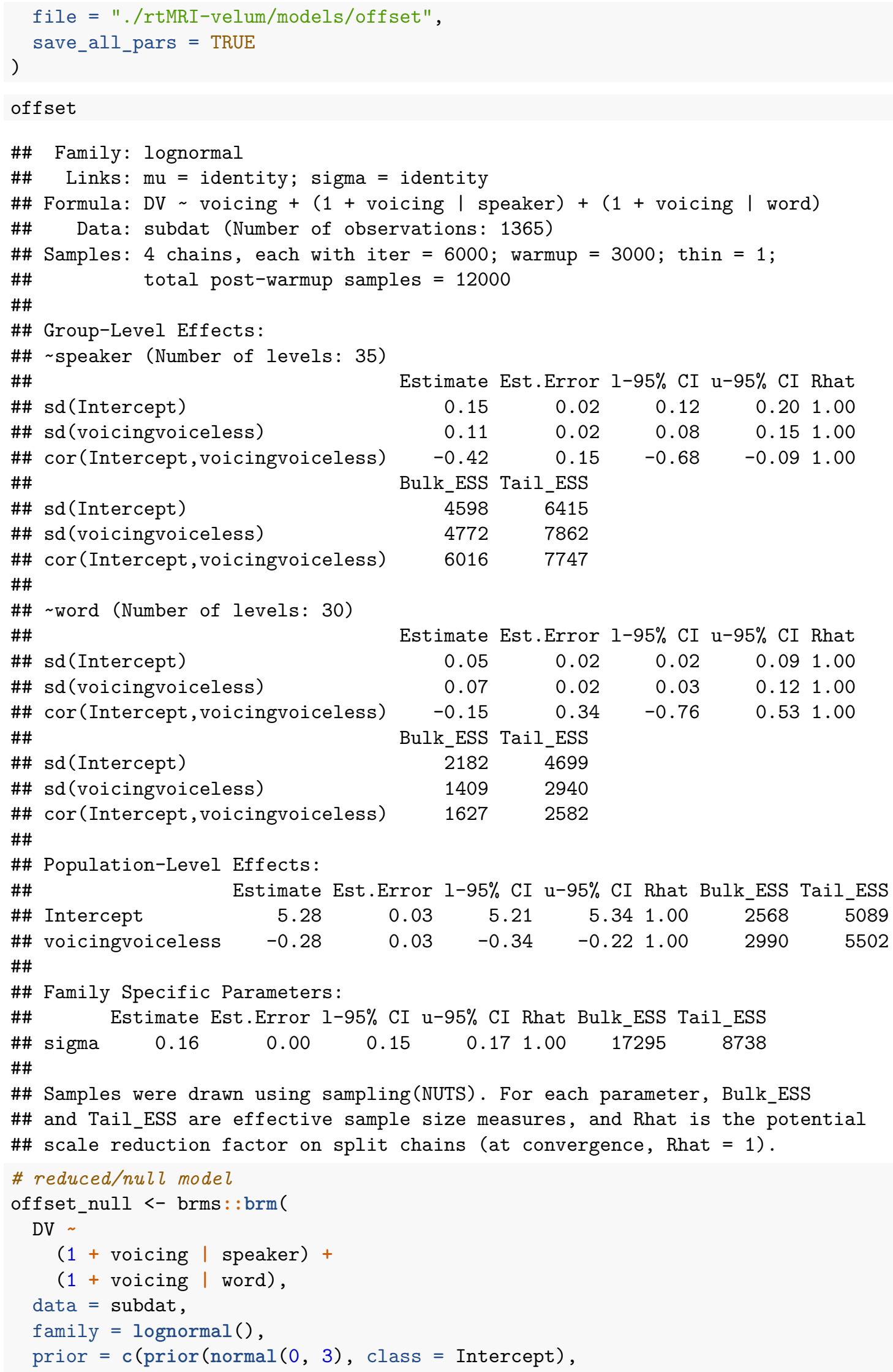




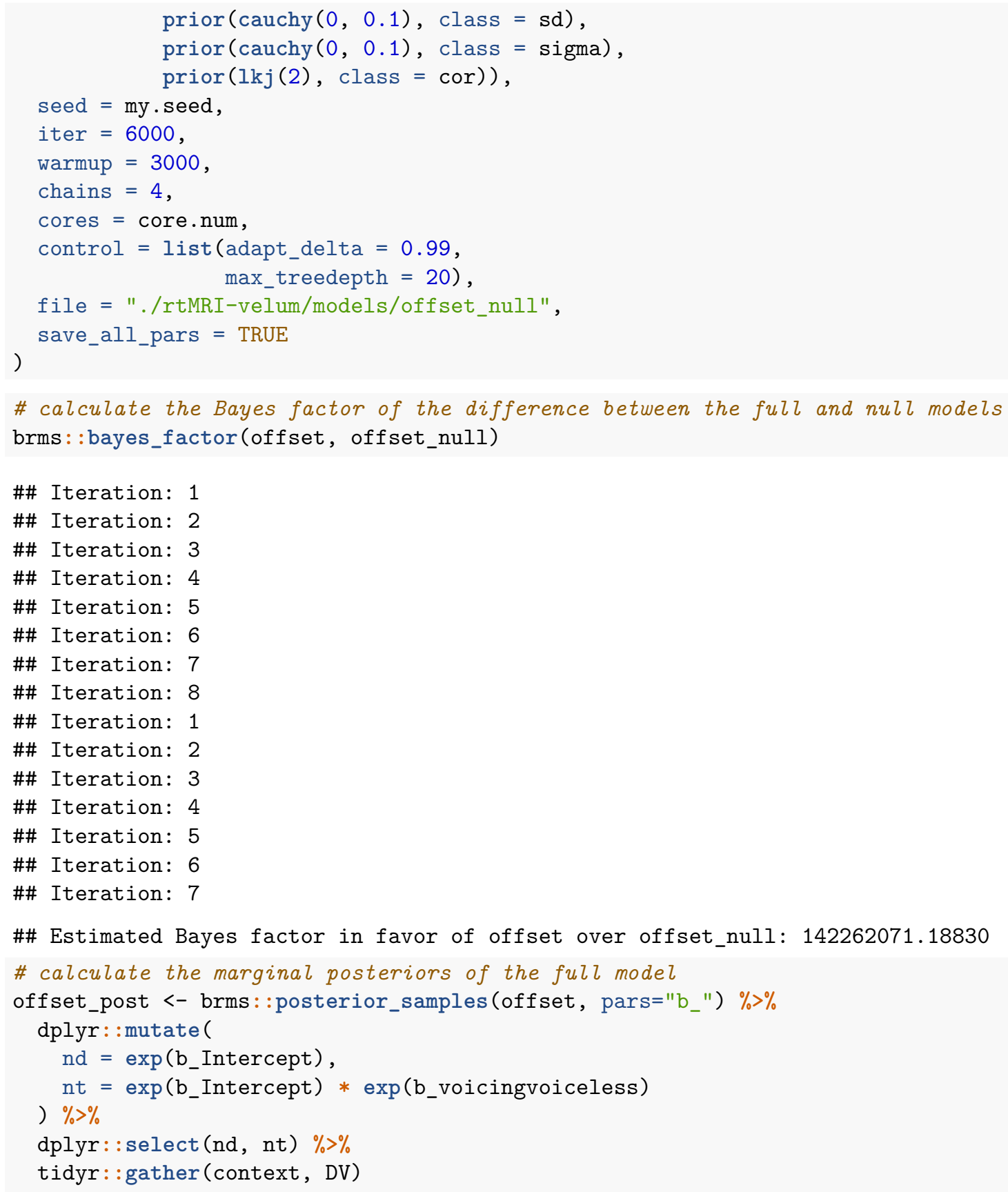

\subsection{Checks}

pp_check (offset, nsamples $=50)+$ theme_minimal $($ ) 


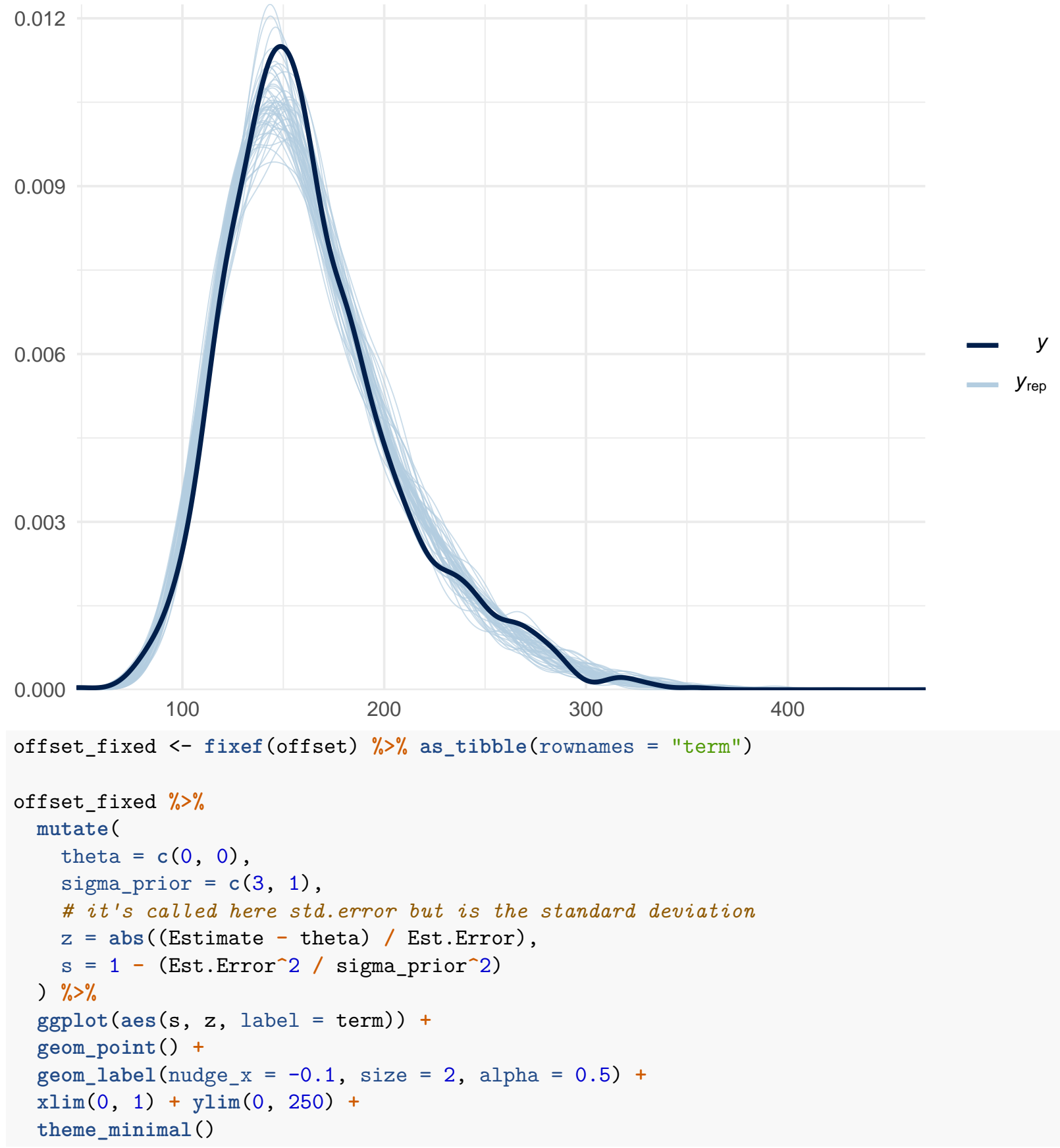




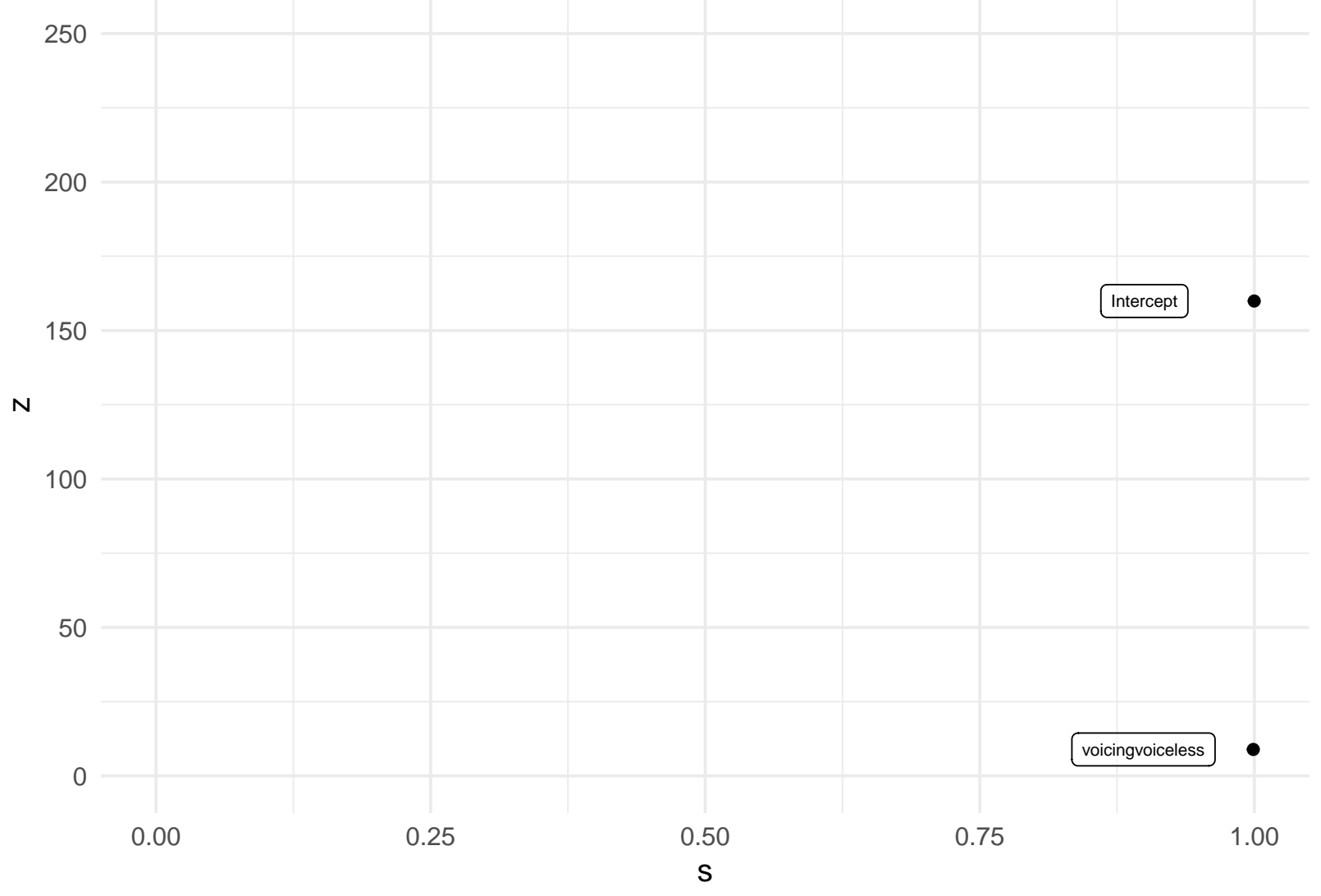

\section{Velum gesture peak (magnitude)}

The BRM for the velum gesture magnitude was built using a Beta distribution, since the magnitude values are on a $0-1$ scale. The following practically flat priors were used: $\operatorname{Normal}(0,10)$ for the intercept; $\operatorname{Normal}(0,5)$ for voicing and the random effects standard deviations; the brms default prior for the $\phi$ parameter of the beta distribution $(\operatorname{gamma}(0.01,0.01))$; and $L K J(2)$ prior for the random effects correlation.

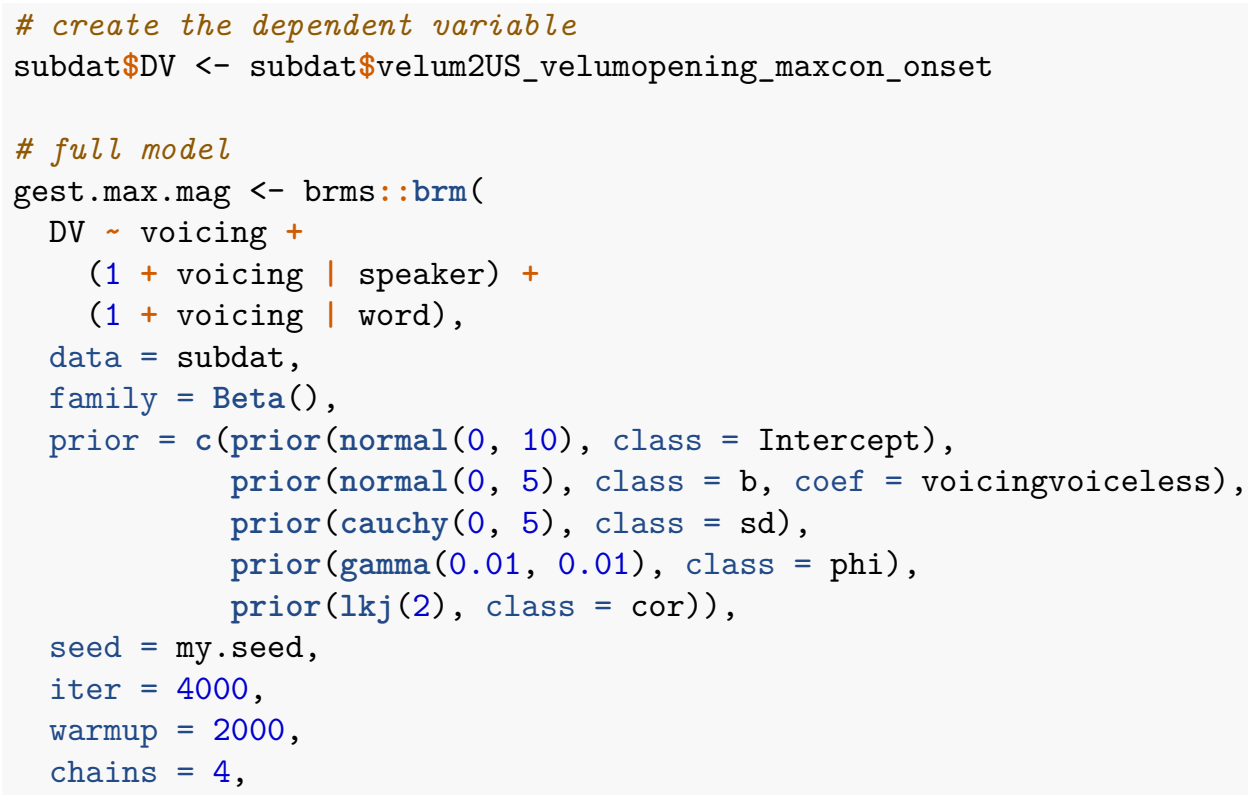




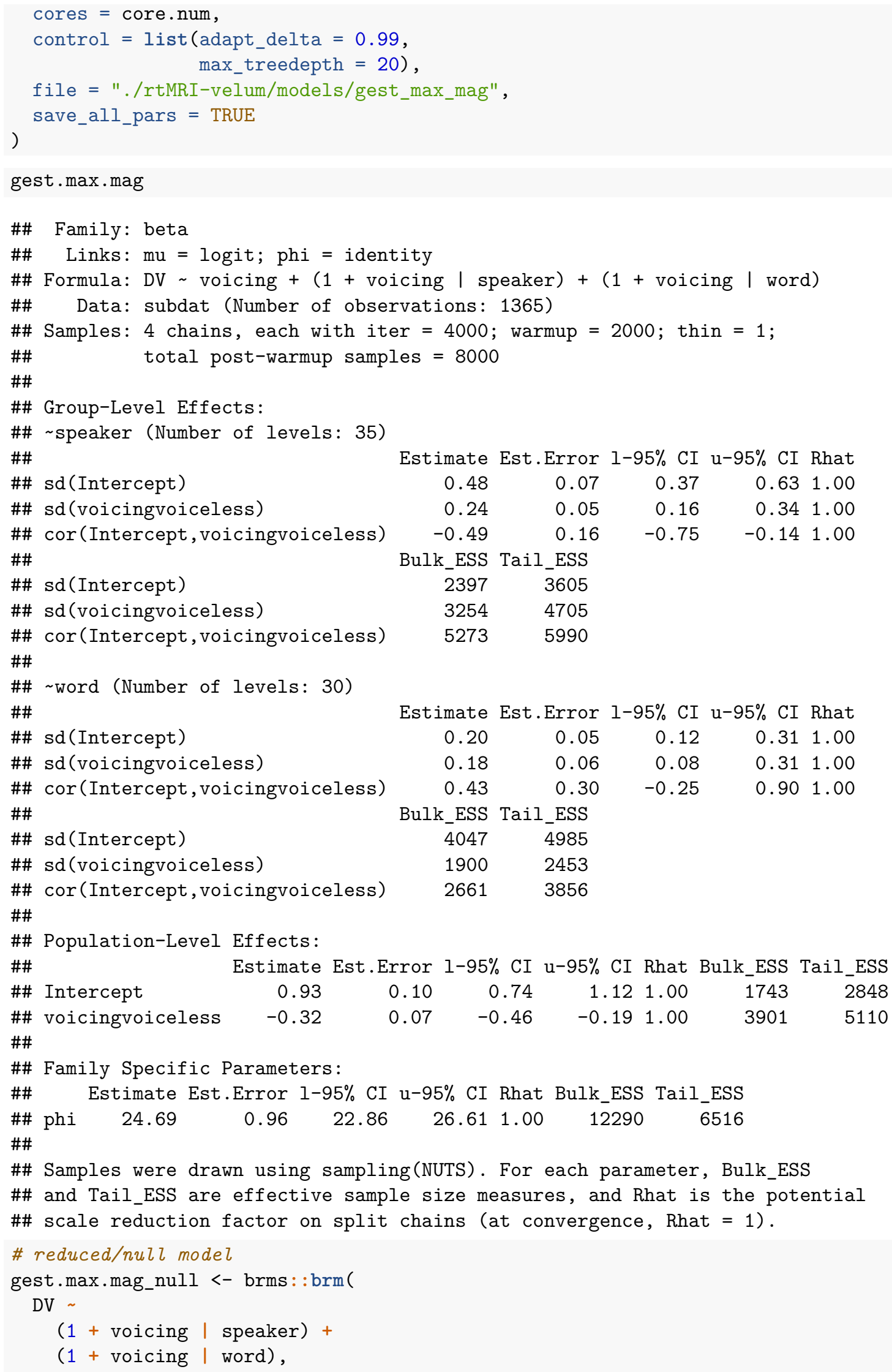




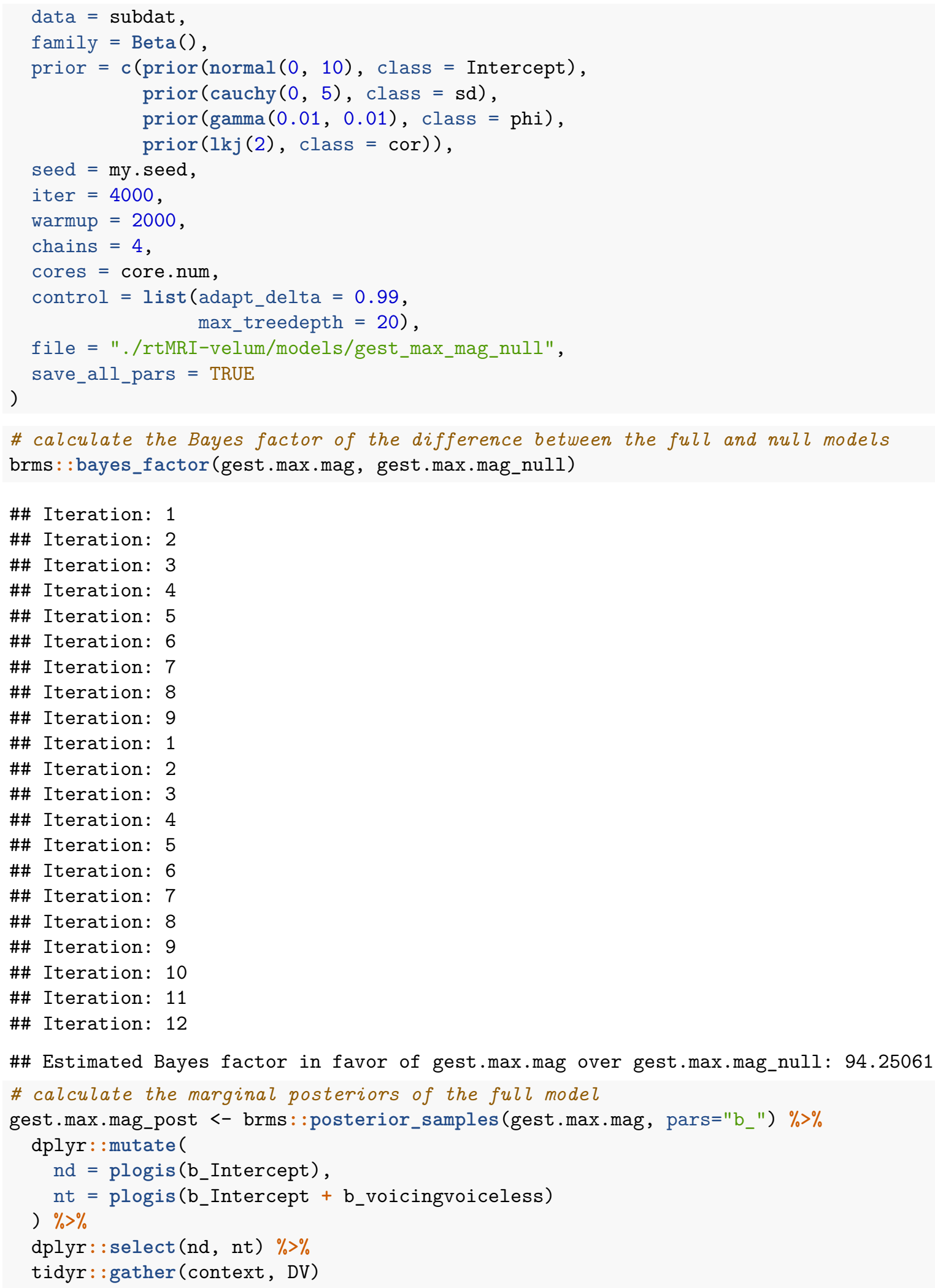




\subsection{Checks}

pp_check (gest.max.mag, nsamples $=50)+$ theme_minimal ()
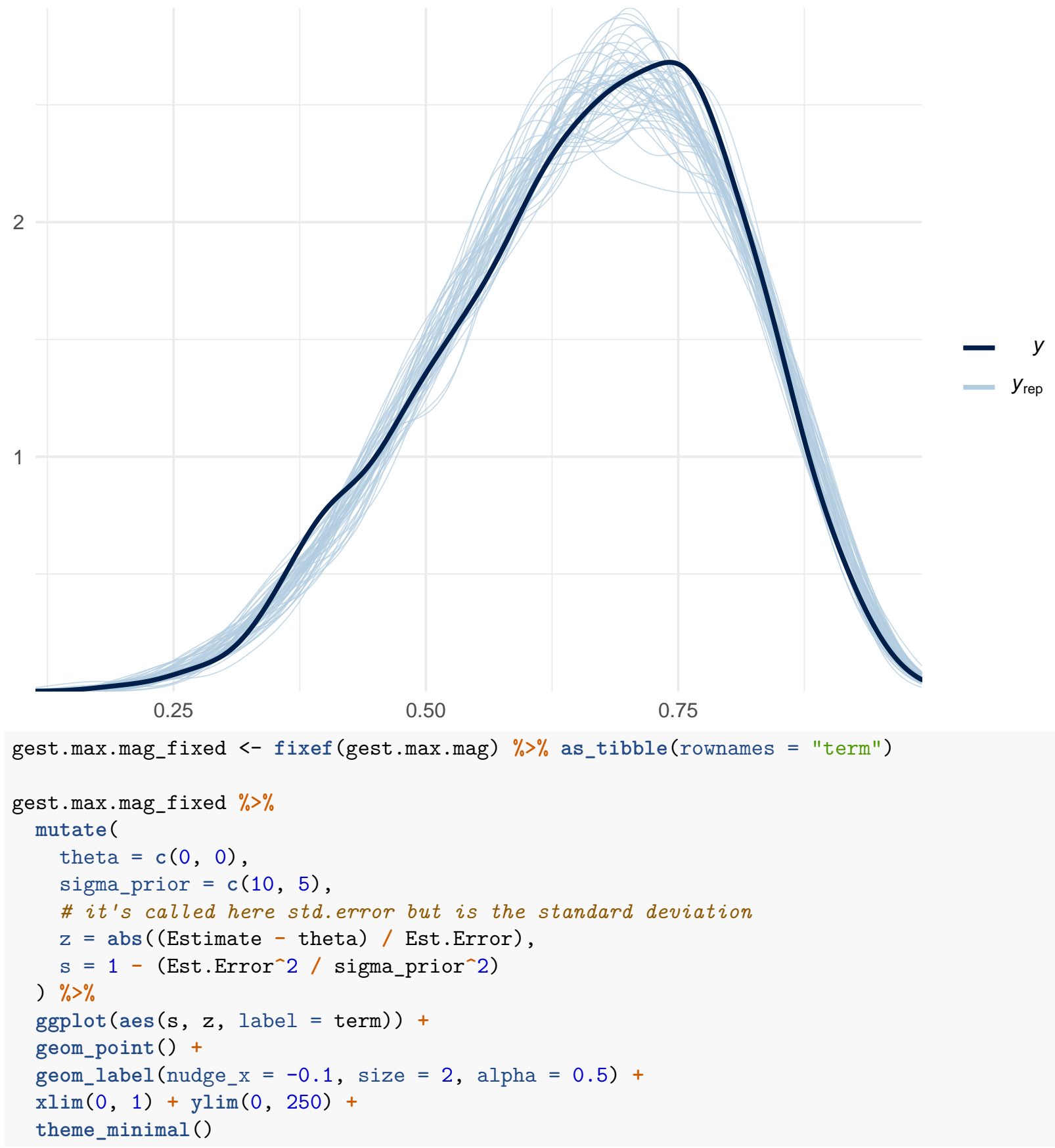


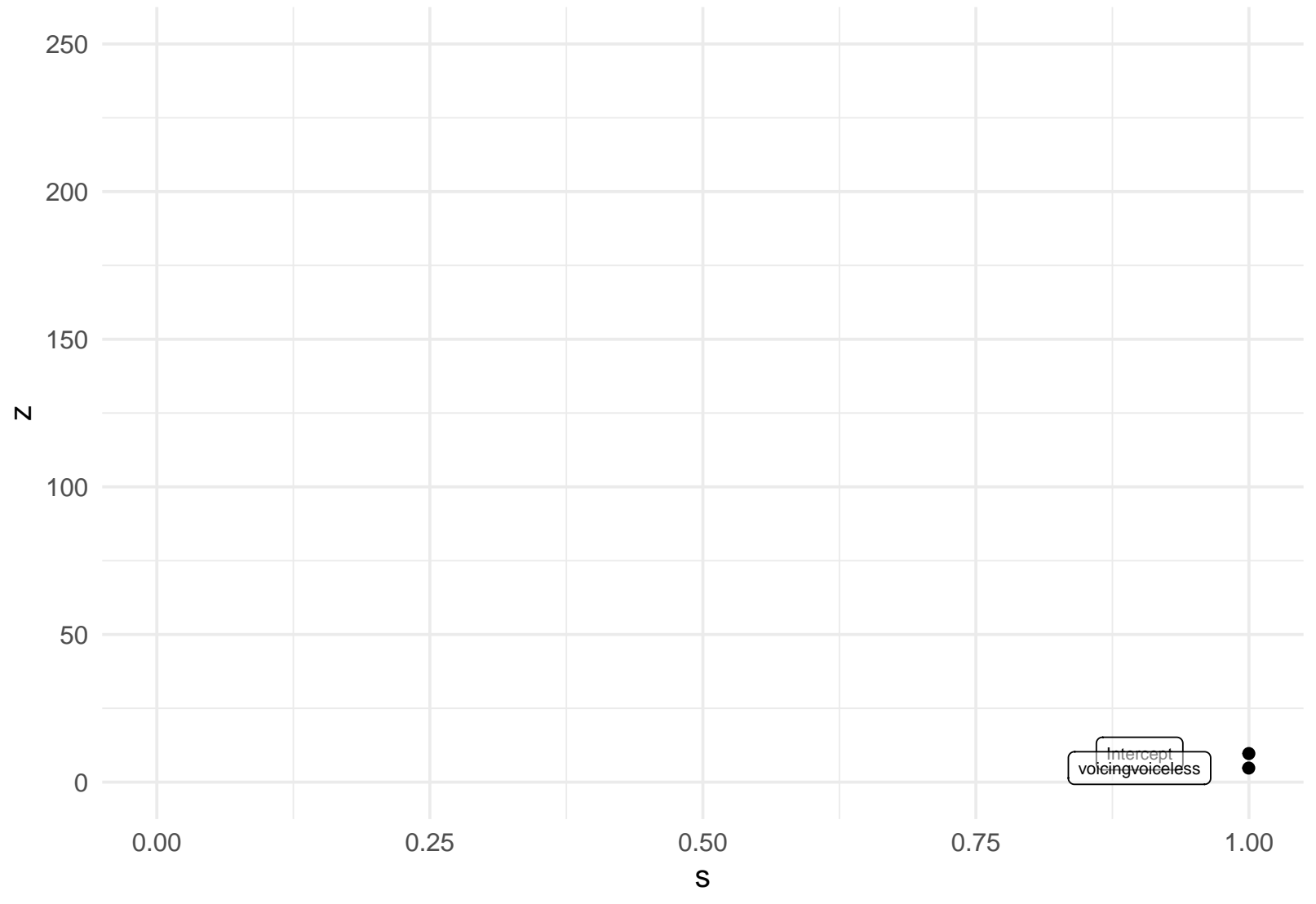

\section{$7 \quad$ Gesture offset stiffness}

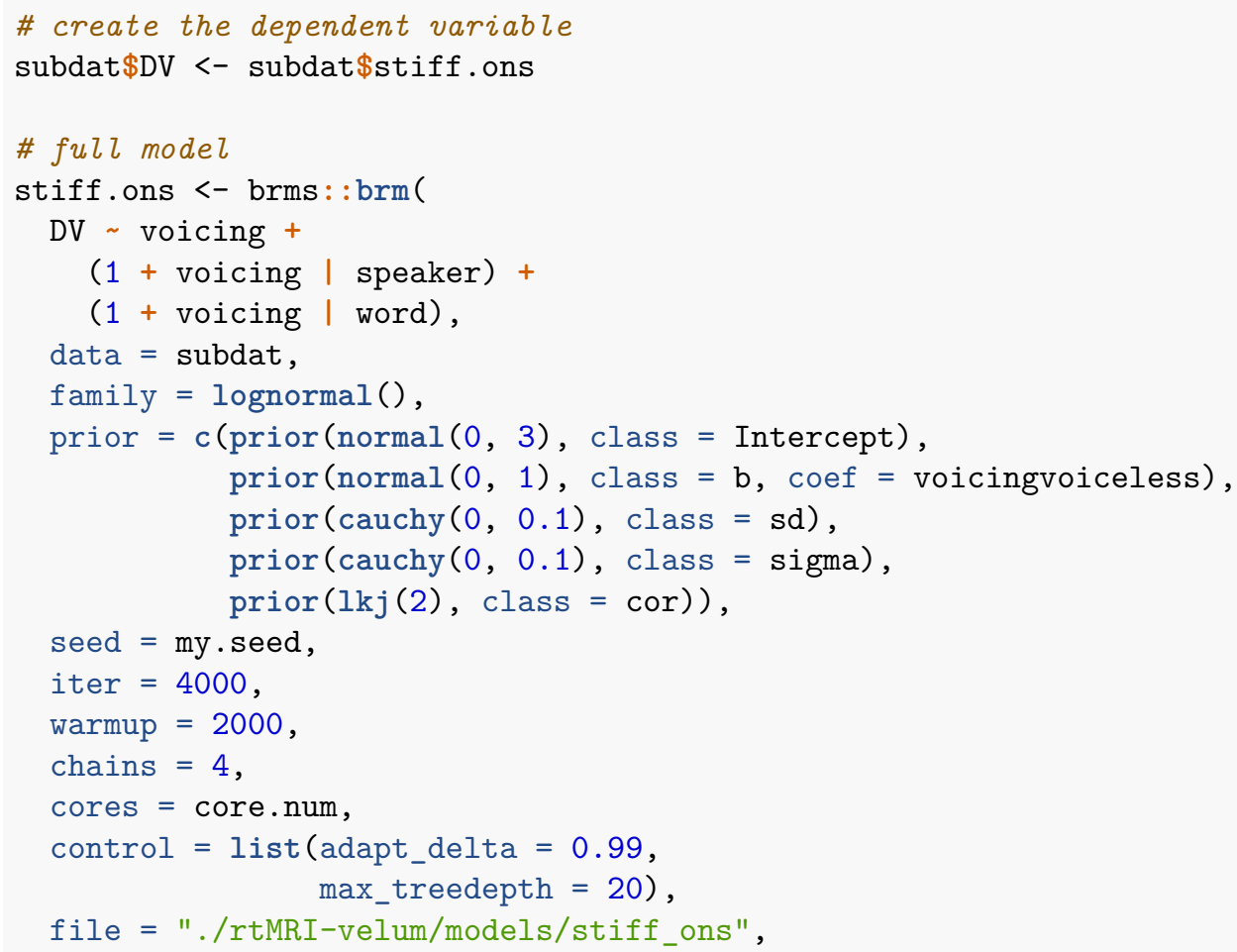




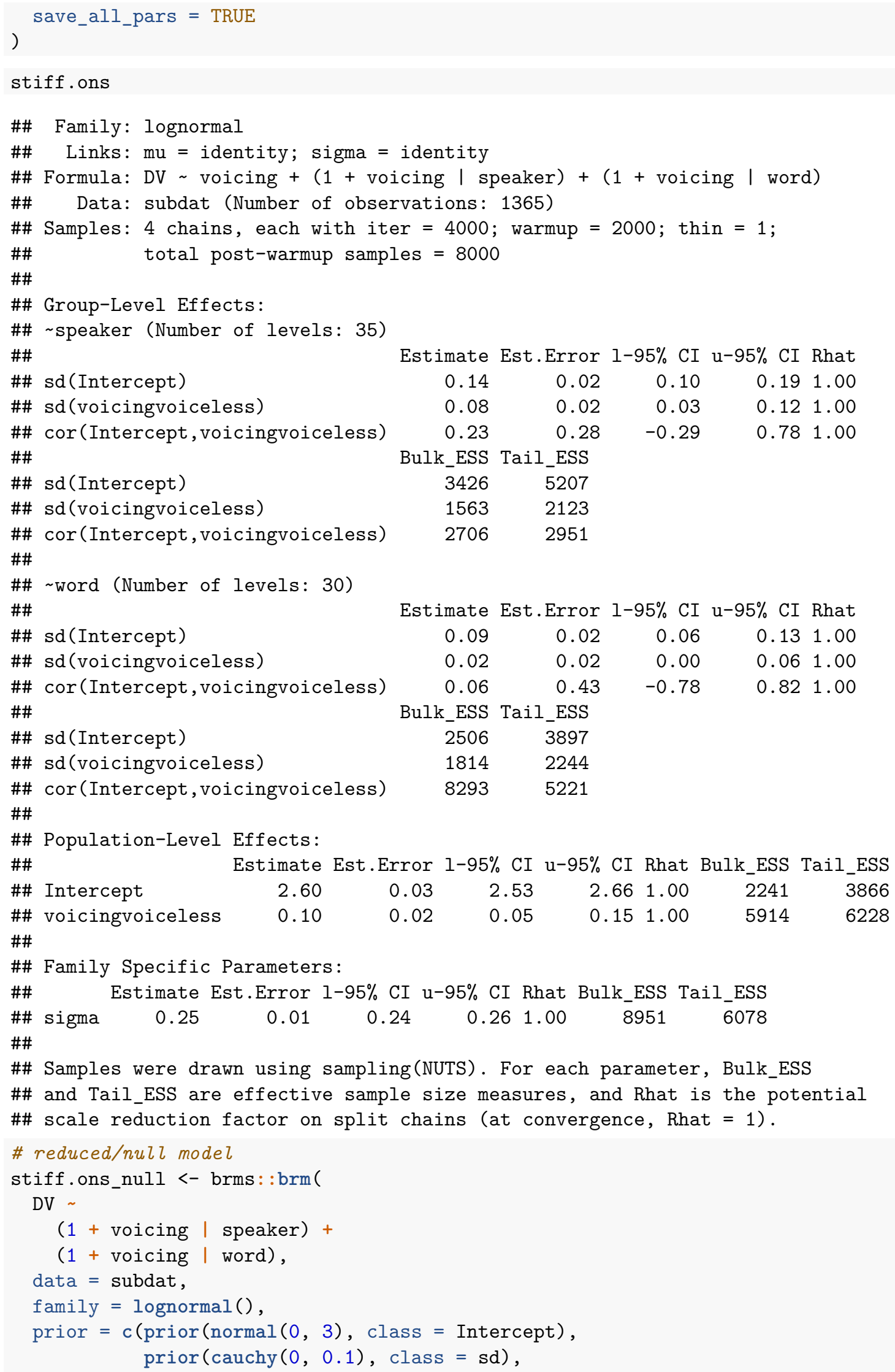




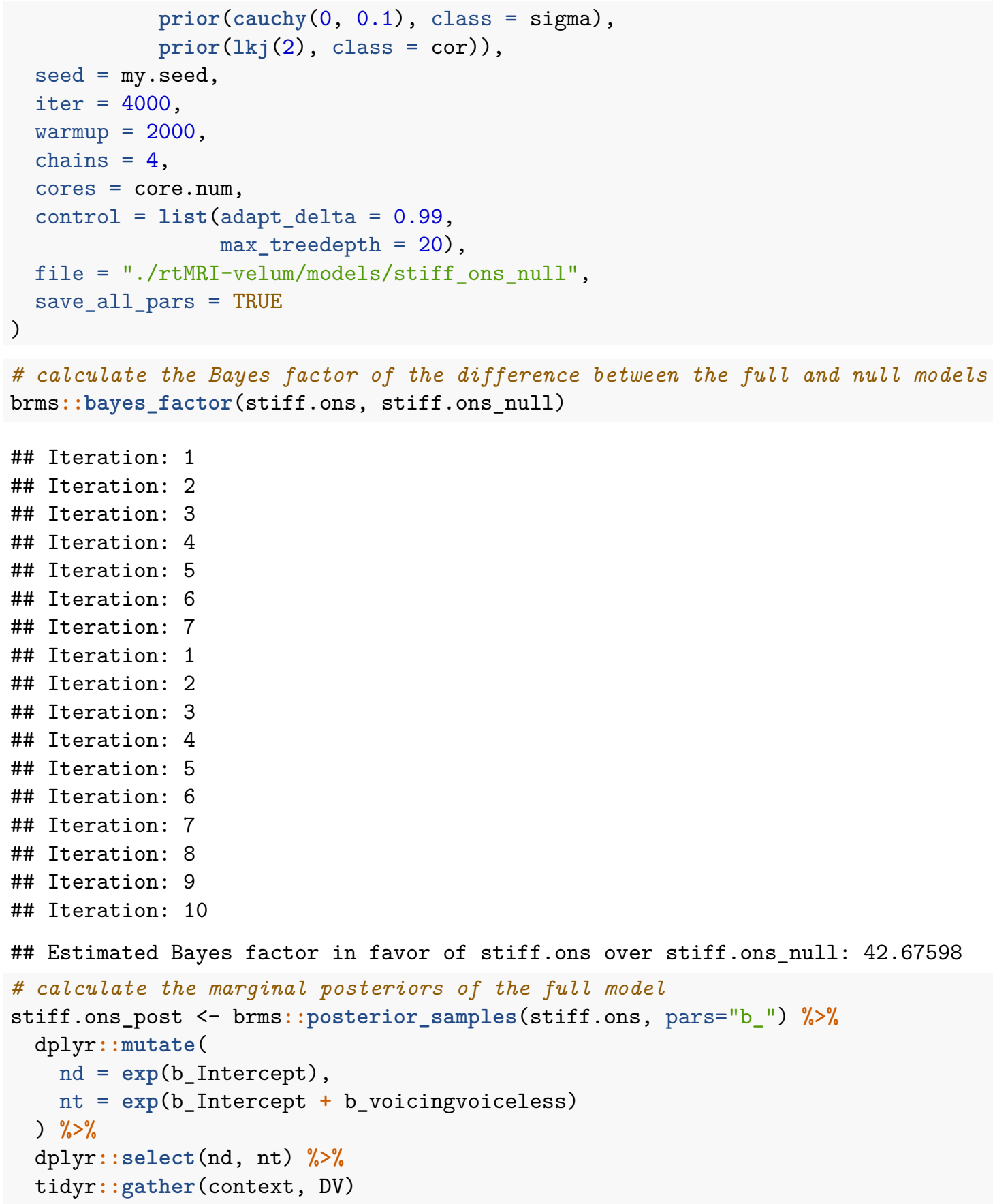

\subsection{Checks}

pp_check(stiff.ons, nsamples $=50)+$ theme_minimal () 


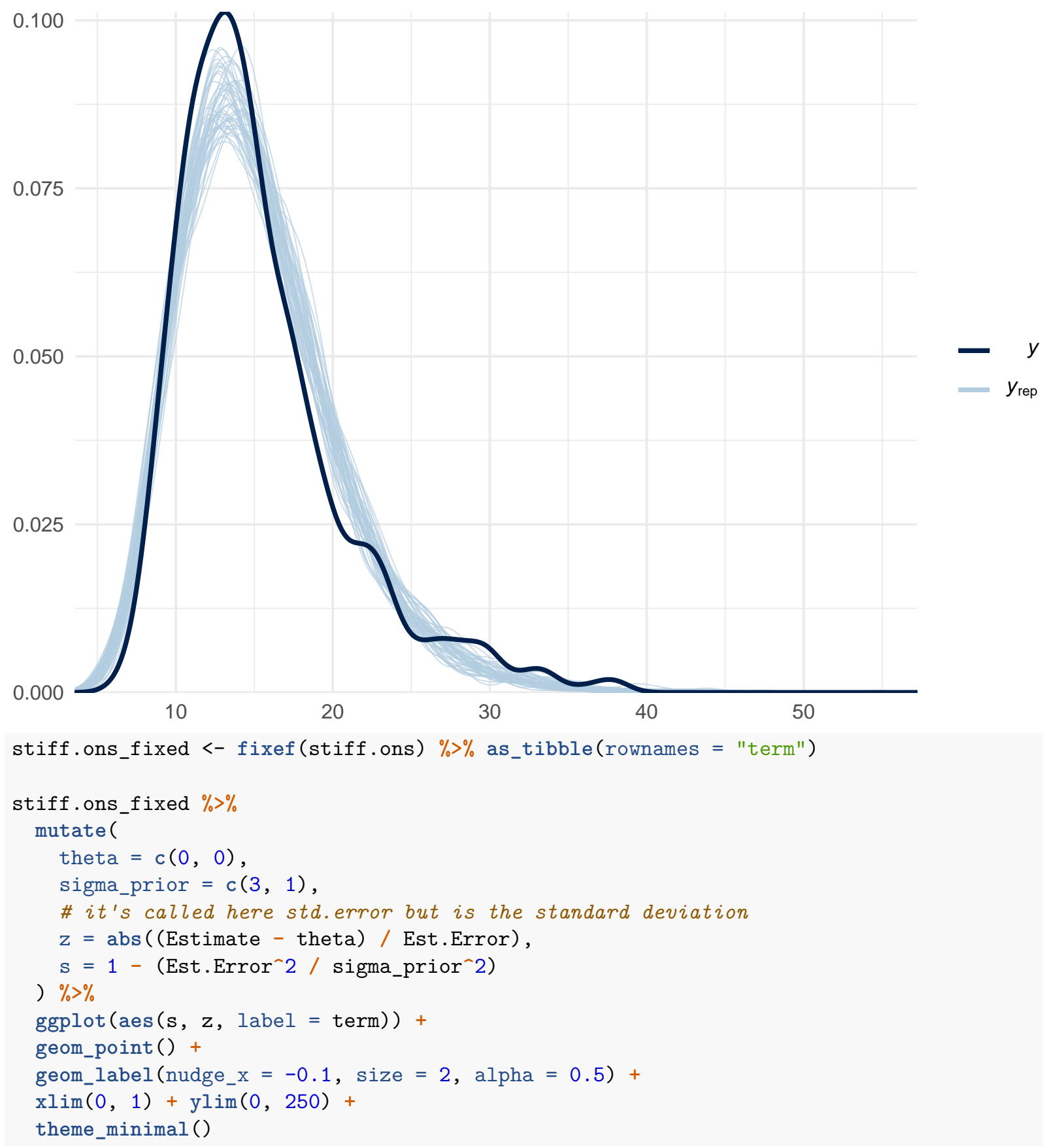




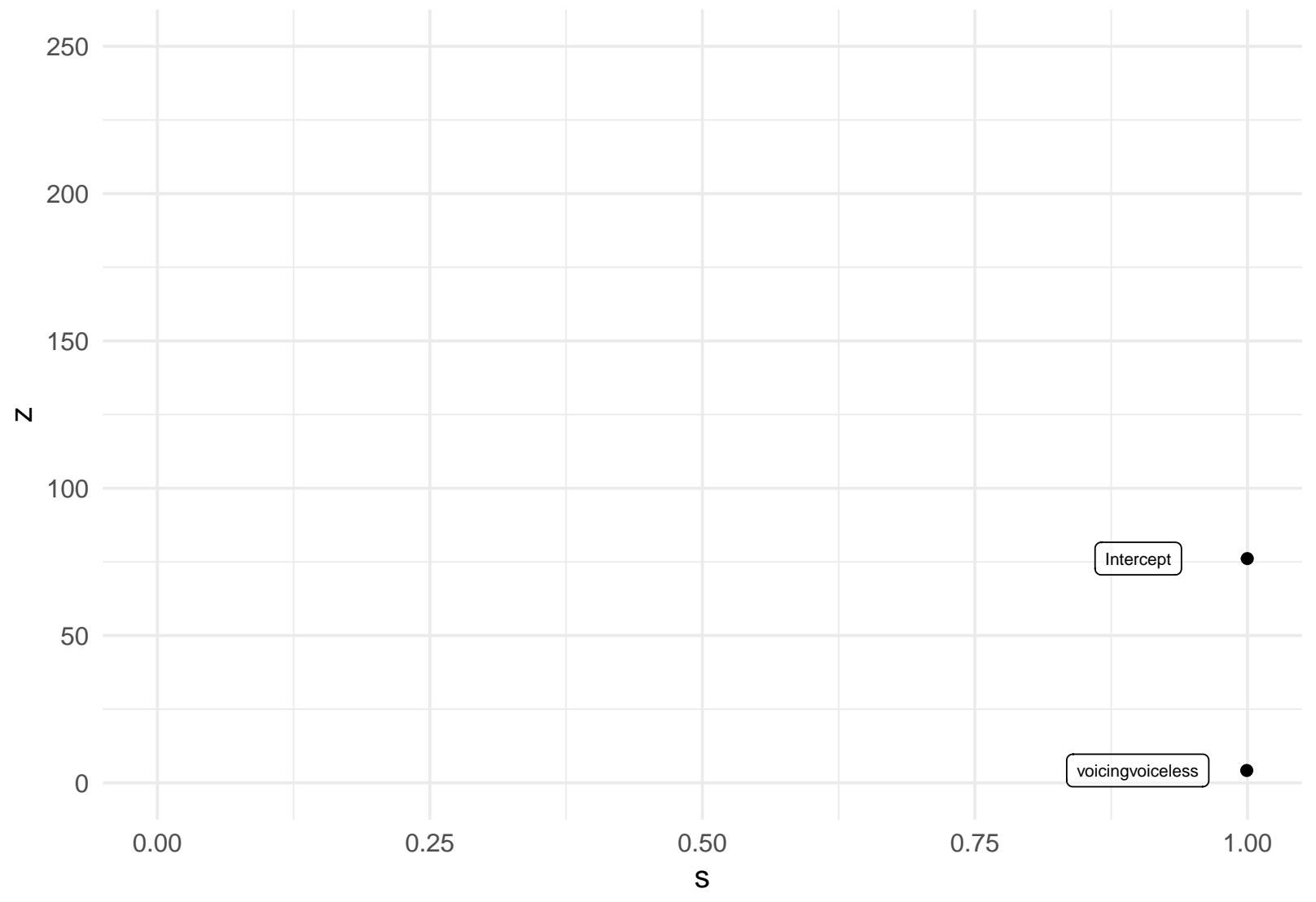

\section{Gesture offset stiffness}

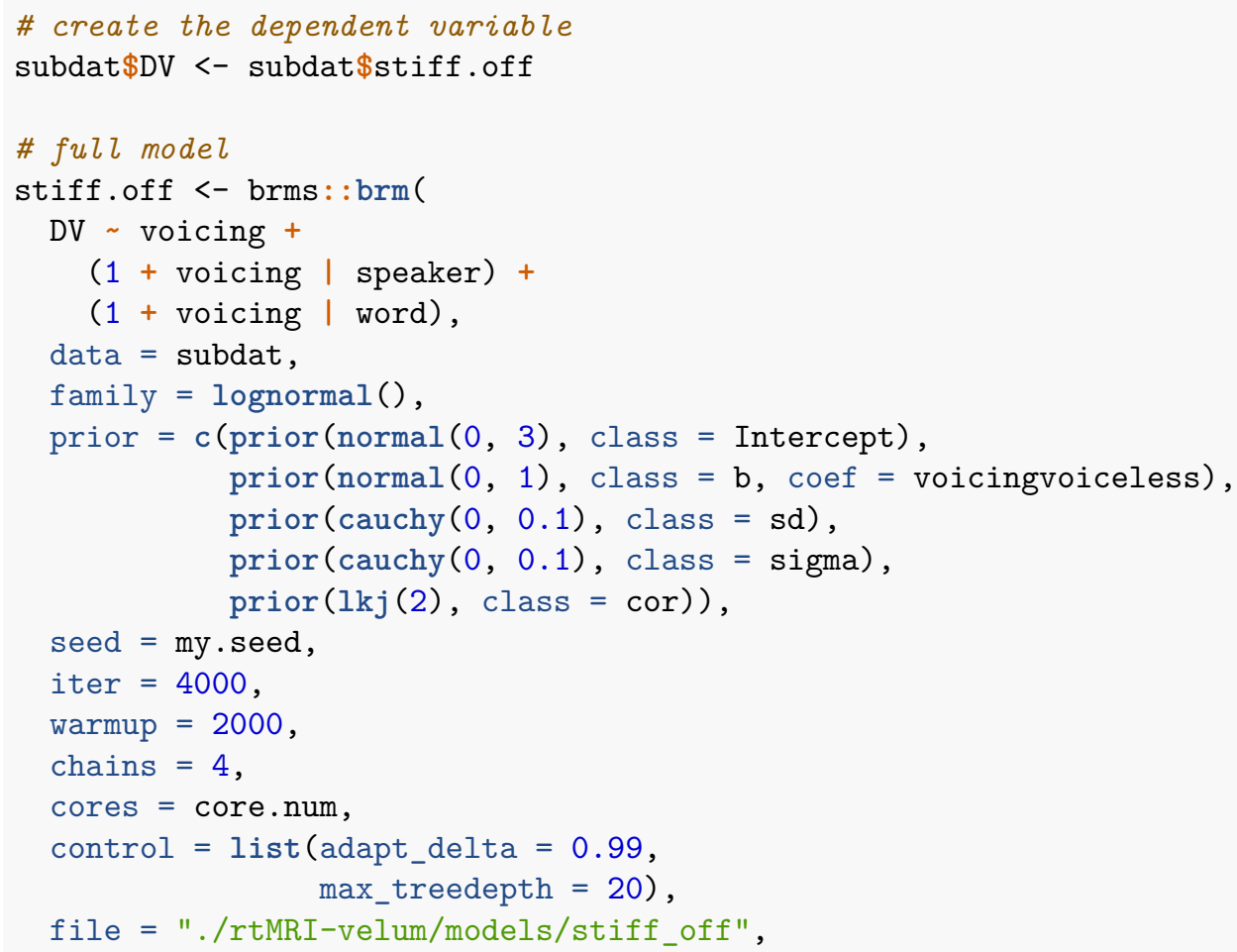




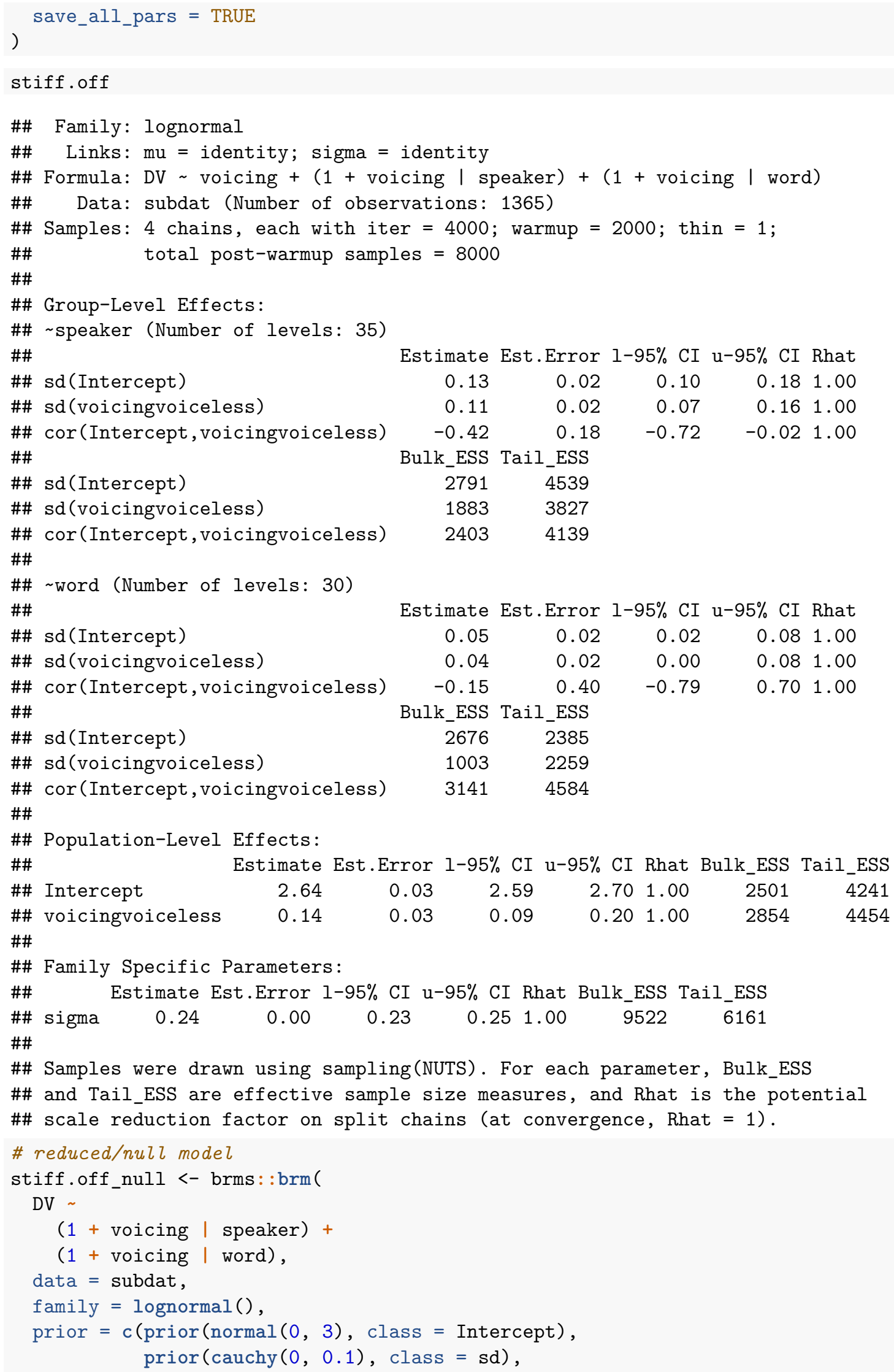




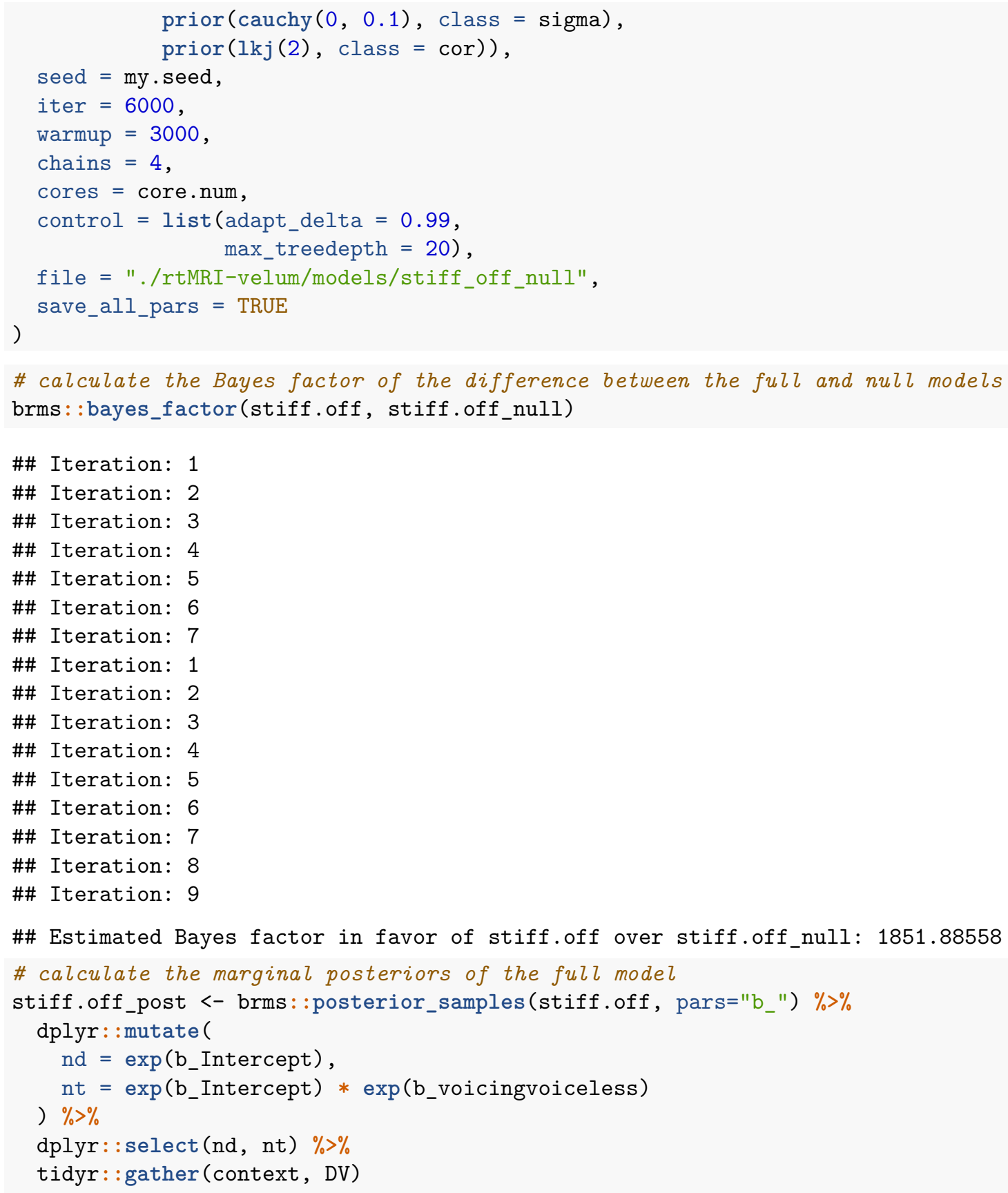

\subsection{Checks}

pp_check(stiff.off, nsamples $=50)+$ theme_minimal $($ ) 


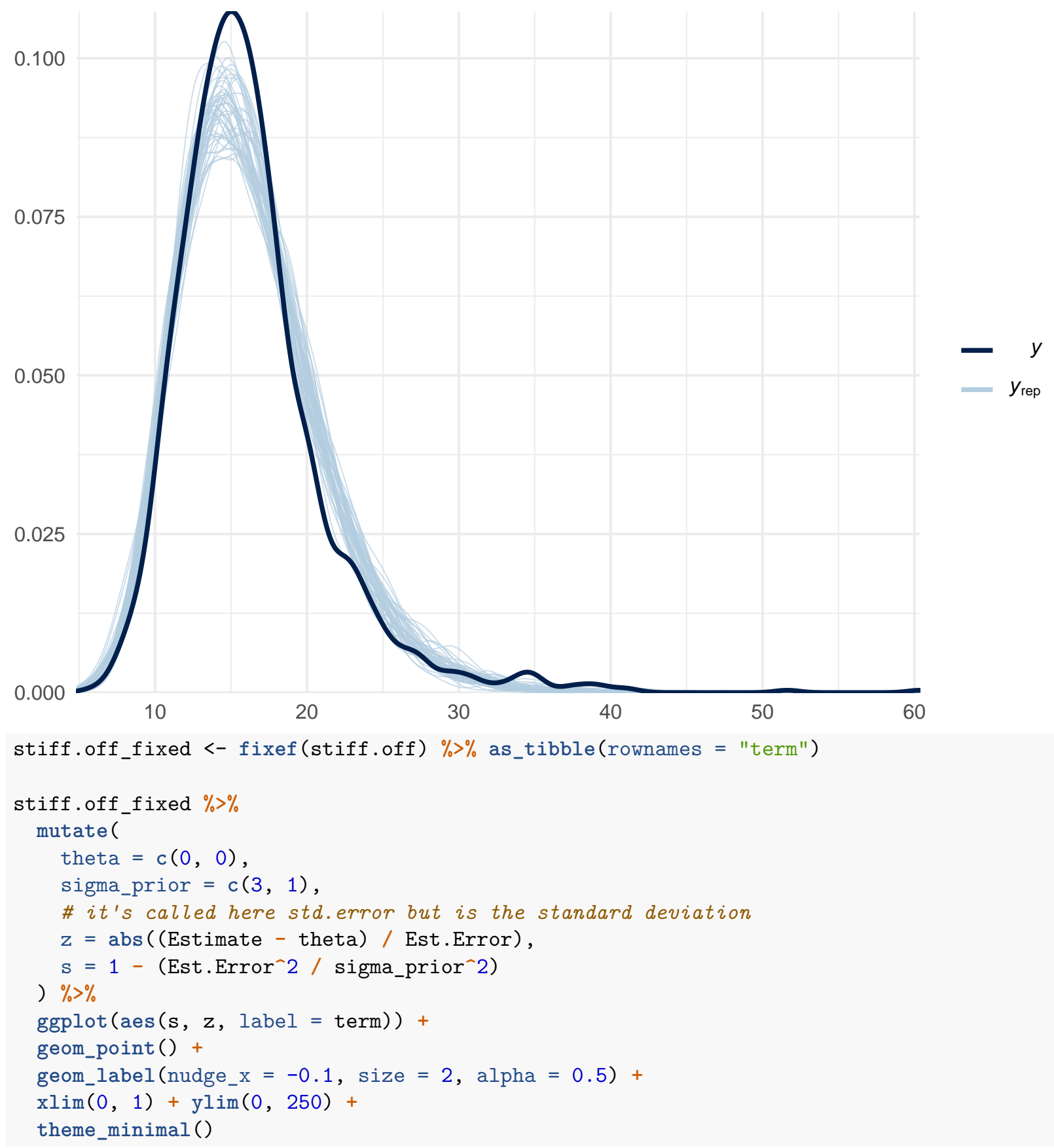




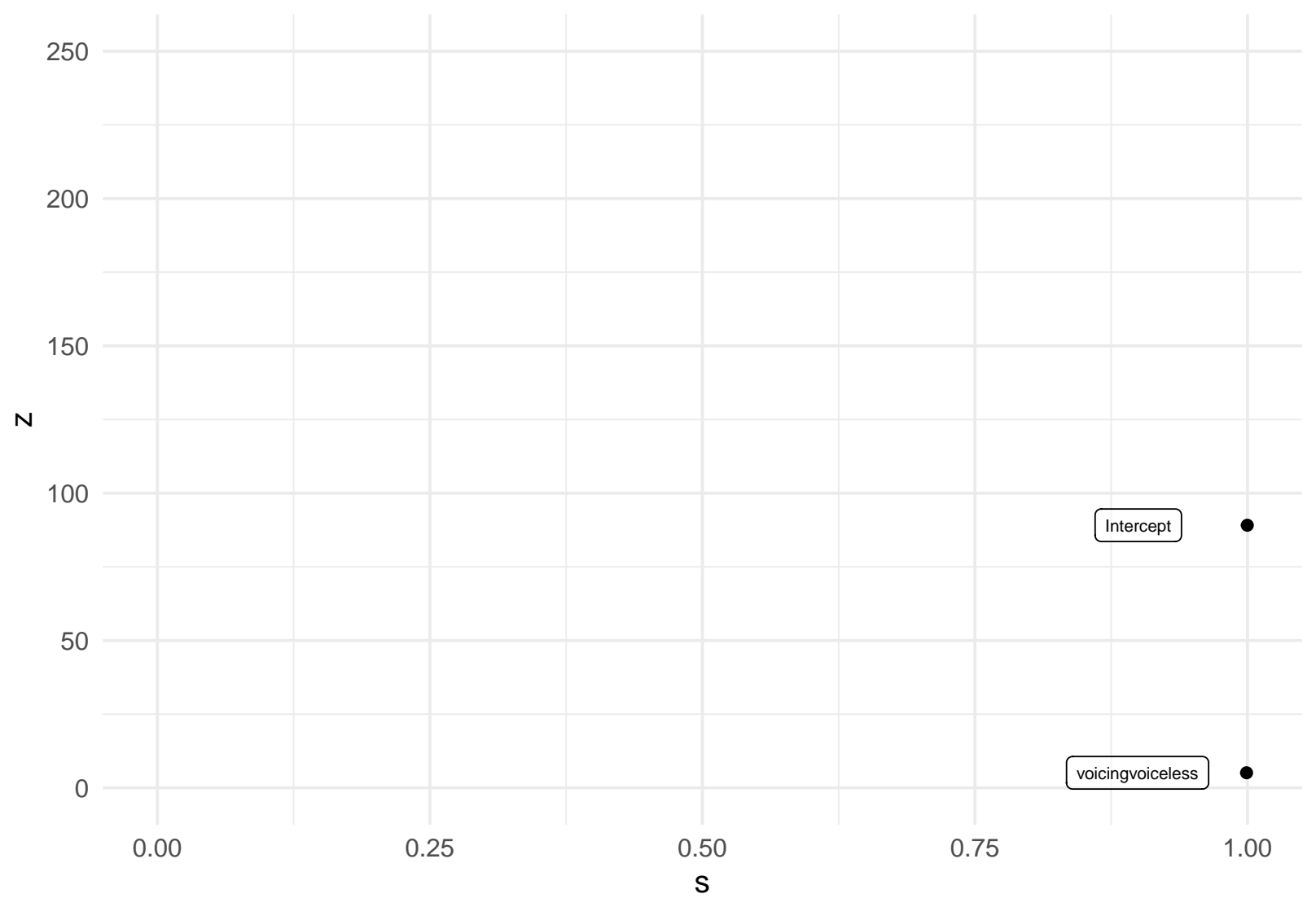

\section{Kurtosis}

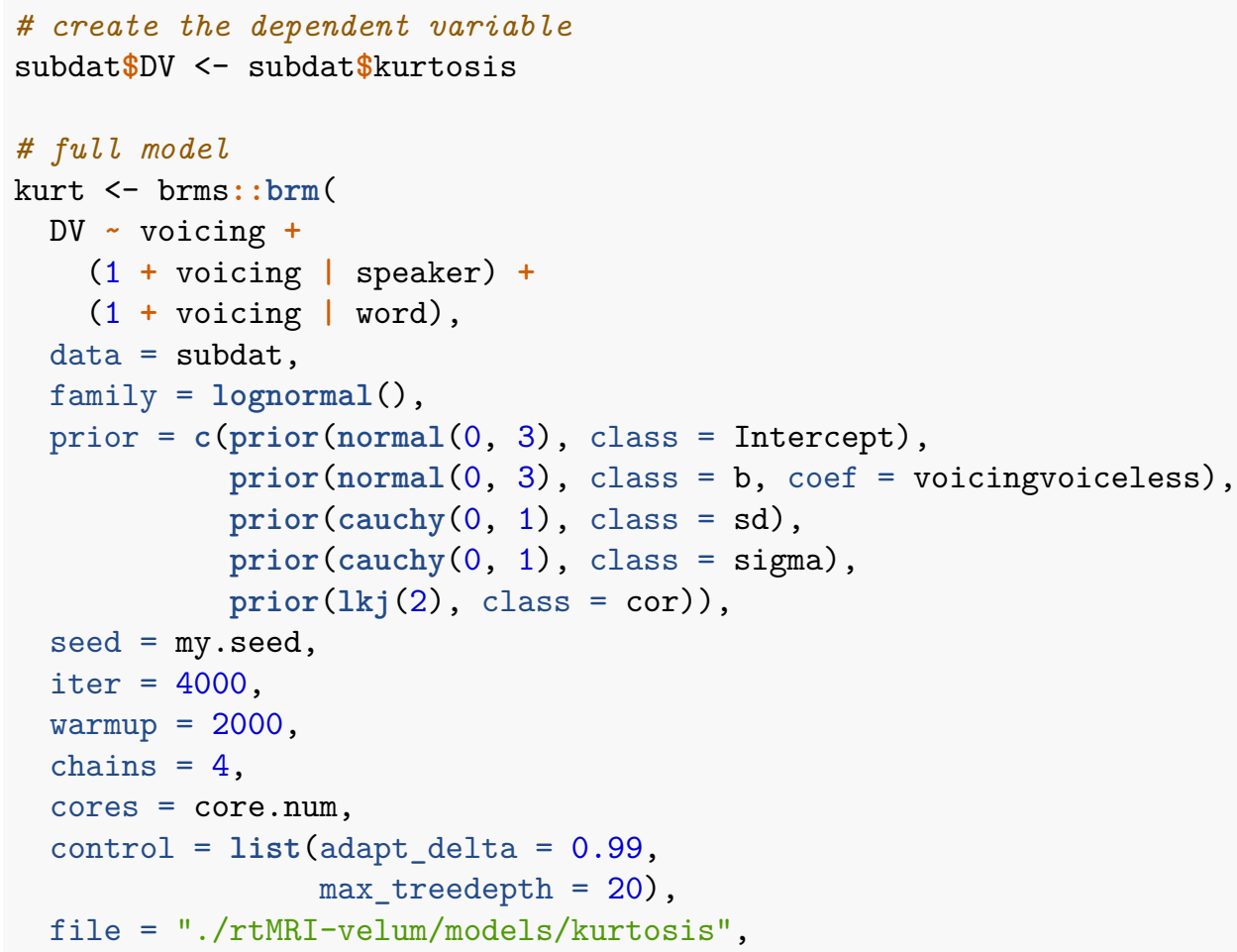




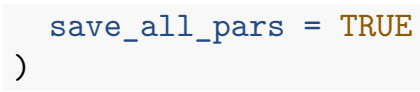

kurt

\#\# Family: lognormal

\#\# Links: $\mathrm{mu}=$ identity; sigma = identity

\#\# Formula: DV voicing + $(1+$ voicing | speaker $)+(1+$ voicing | word $)$

\#\# Data: subdat (Number of observations: 1365)

\#\# Samples: 4 chains, each with iter $=4000$; warmup $=2000$; thin $=1$;

\#\# total post-warmup samples $=8000$

\#\#

\#\# Group-Level Effects:

\#\# speaker (Number of levels: 35)

\#\#

\#\# sd(Intercept)

\#\# sd(voicingvoiceless)

\#\# cor(Intercept,voicingvoiceless)

\#\#

\#\# sd(Intercept)

\#\# sd(voicingvoiceless)

Estimate Est.Error 1-95\% CI u-95\% CI Rhat

\#\#

\#\# word (Number of levels: 30 )

\#\#

\#\# sd(Intercept)

\#\# sd(voicingvoiceless)

$\begin{array}{lllll}0.07 & 0.01 & 0.04 & 0.09 & 1.00\end{array}$

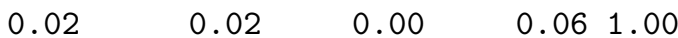

$\begin{array}{ll}-0.11 & 0.42\end{array}$

$-0.81$

0.741 .00

\#\# cor(Intercept, voicingvoiceless)

\#\#

\#\# sd(Intercept)

Bulk_ESS Tail_ESS

$3293 \quad 4759$

$1573 \quad 3551$

$6016 \quad 5689$

\#\# sd(voicingvoiceless)

\#\# cor(Intercept,voicingvoiceless) $4650 \quad 5020$

\#\#

\#\# Population-Level Effects:

\#\#

Estimate Est.Error 1-95\% CI u-95\% CI Rhat Bulk_ESS Tail_ESS

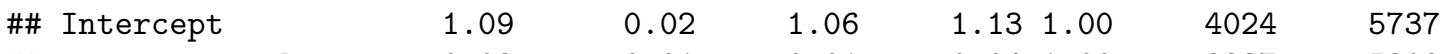

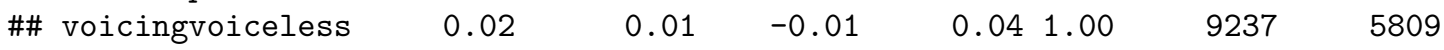

\#\#

\#\# Family Specific Parameters:

\#\# Estimate Est.Error 1-95\% CI u-95\% CI Rhat Bulk_ESS Tail_ESS

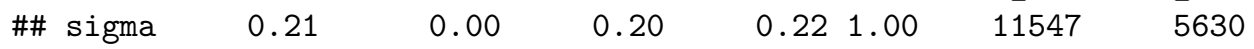

\#\#

\#\# Samples were drawn using sampling(NUTS). For each parameter, Bulk_ESS

\#\# and Tail_ESS are effective sample size measures, and Rhat is the potential

\#\# scale reduction factor on split chains (at convergence, Rhat $=1$ ).

\# reduced/null model

kurt_null <- brms : :brm(

DV

$(1+$ voicing | speaker) +

$(1+$ voicing | word),

data $=$ subdat,

family = lognormal (),

prior $=c($ prior $($ normal $(0,3), \operatorname{class}=$ Intercept $)$, prior $(\operatorname{cauchy}(0,1), \operatorname{class}=\mathbf{s d})$, 


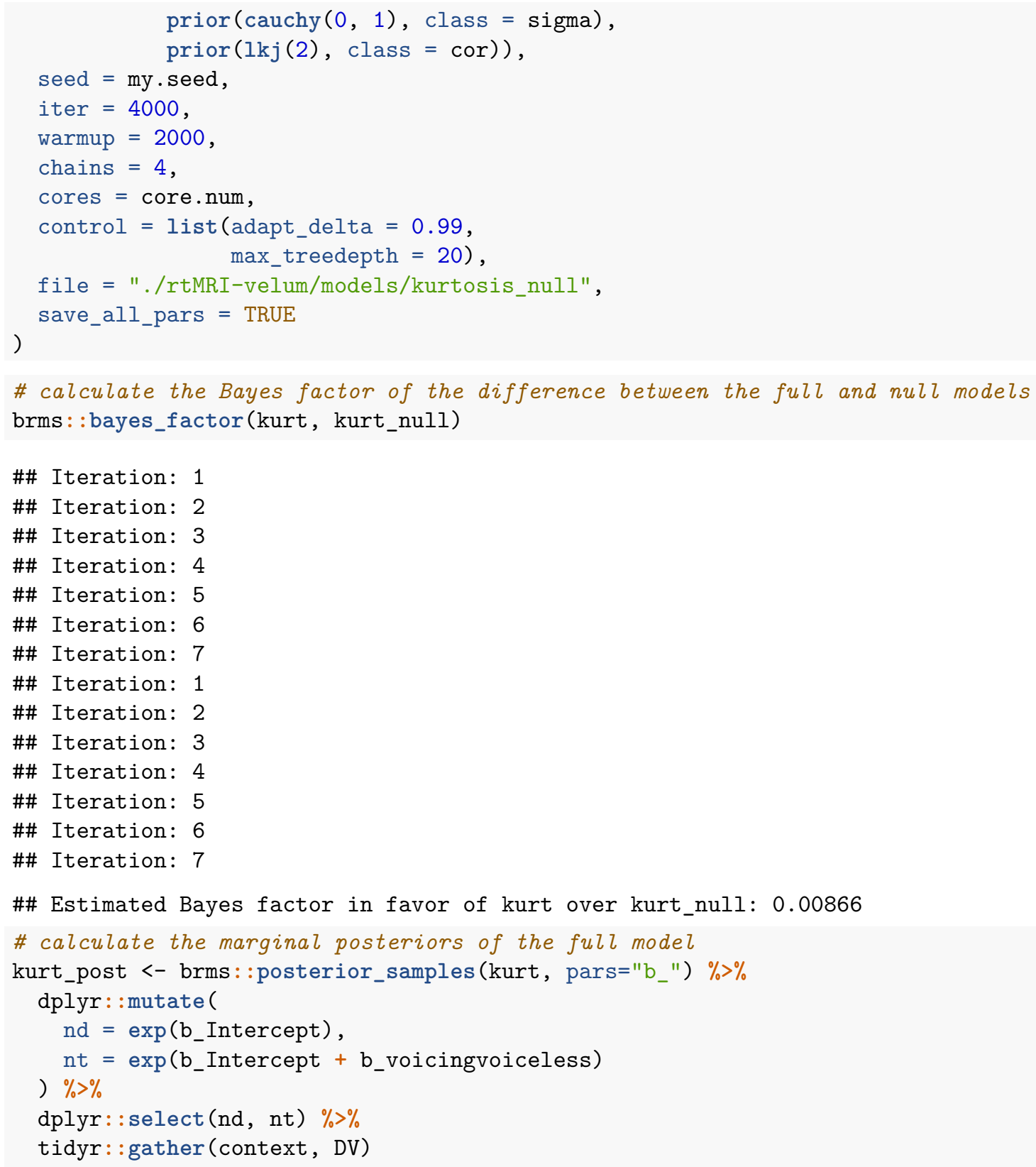

\subsection{Checks}

pp_check(kurt, nsamples $=50)+$ theme_minimal () 


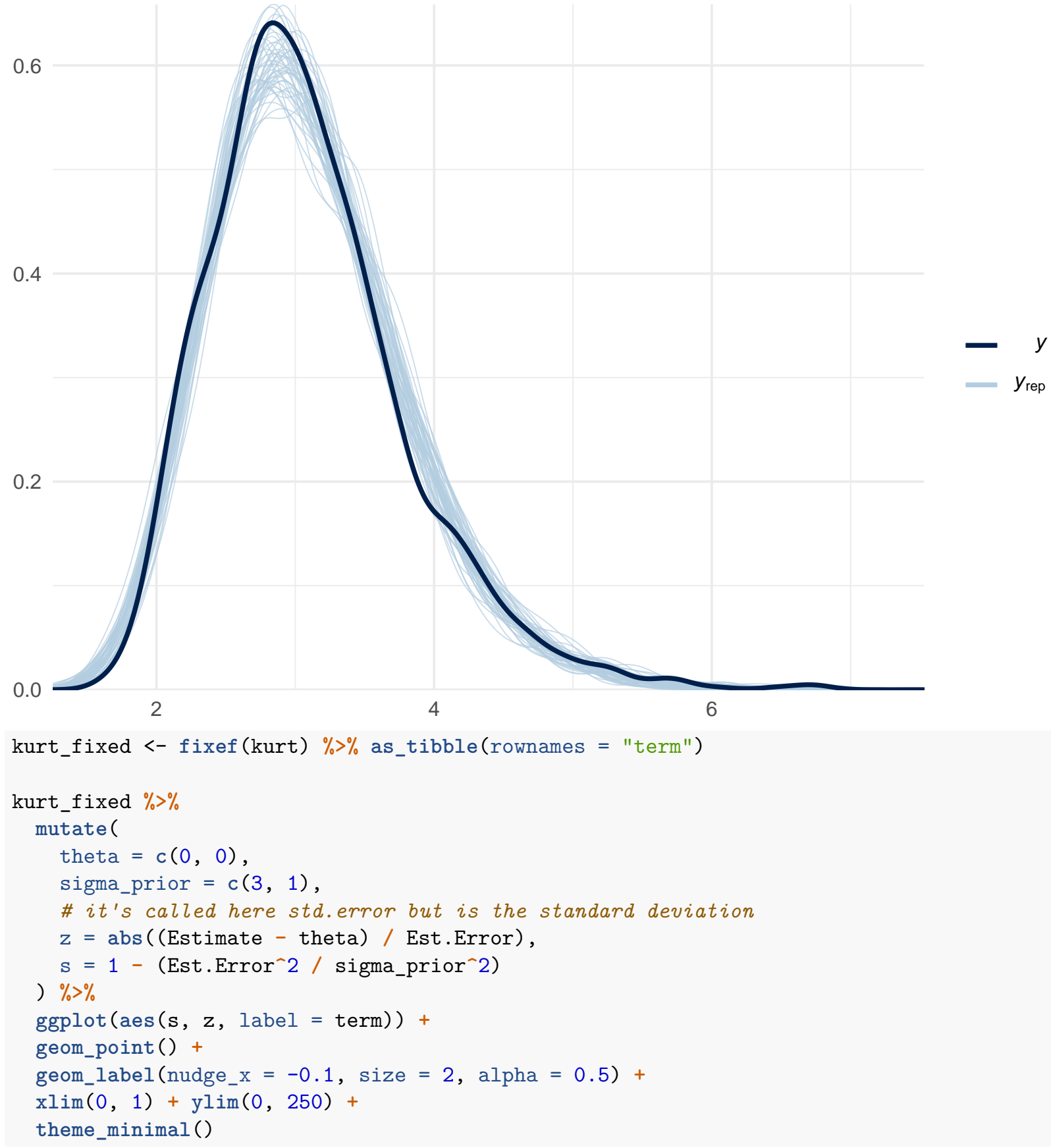




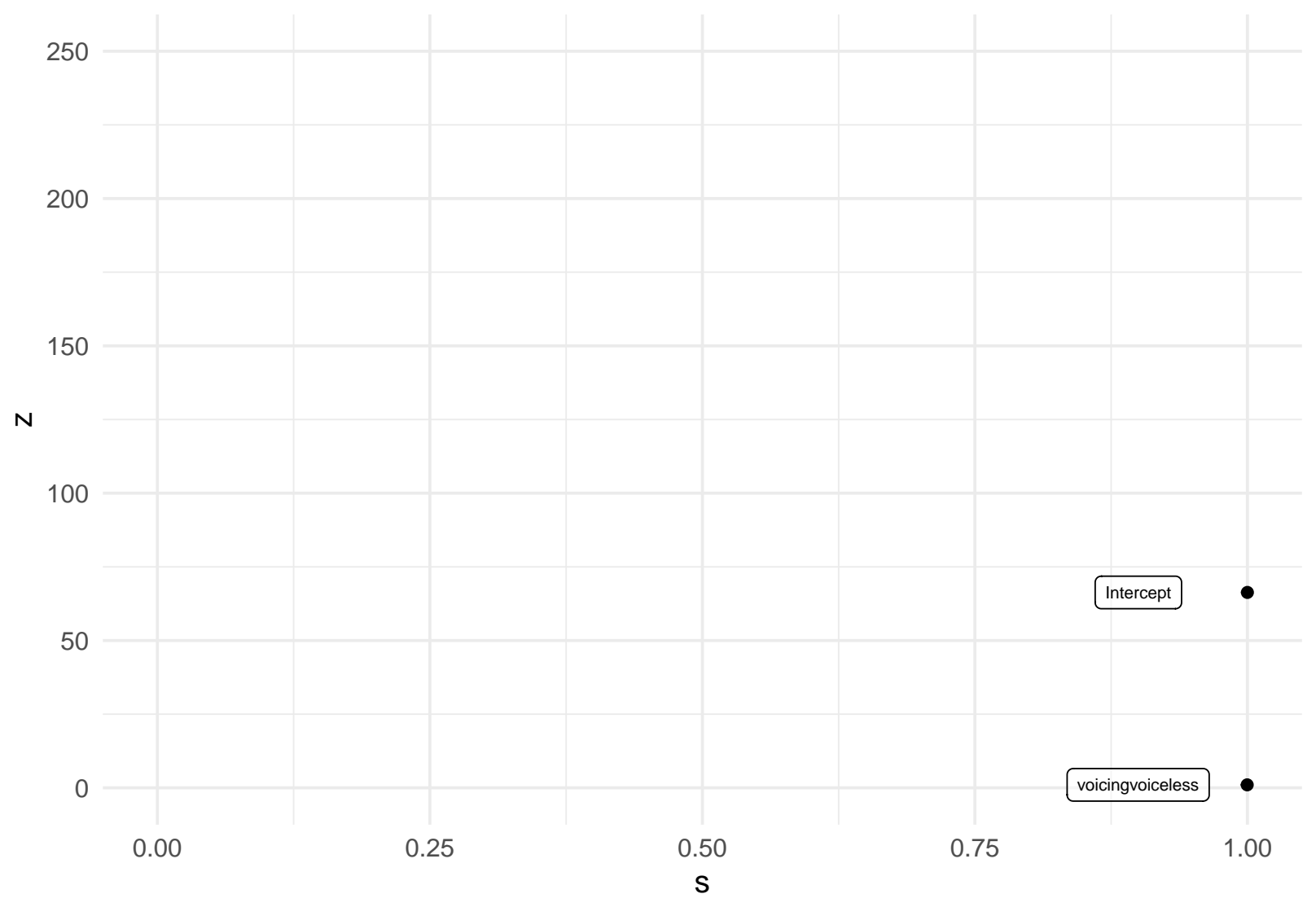

\section{Crest factor}

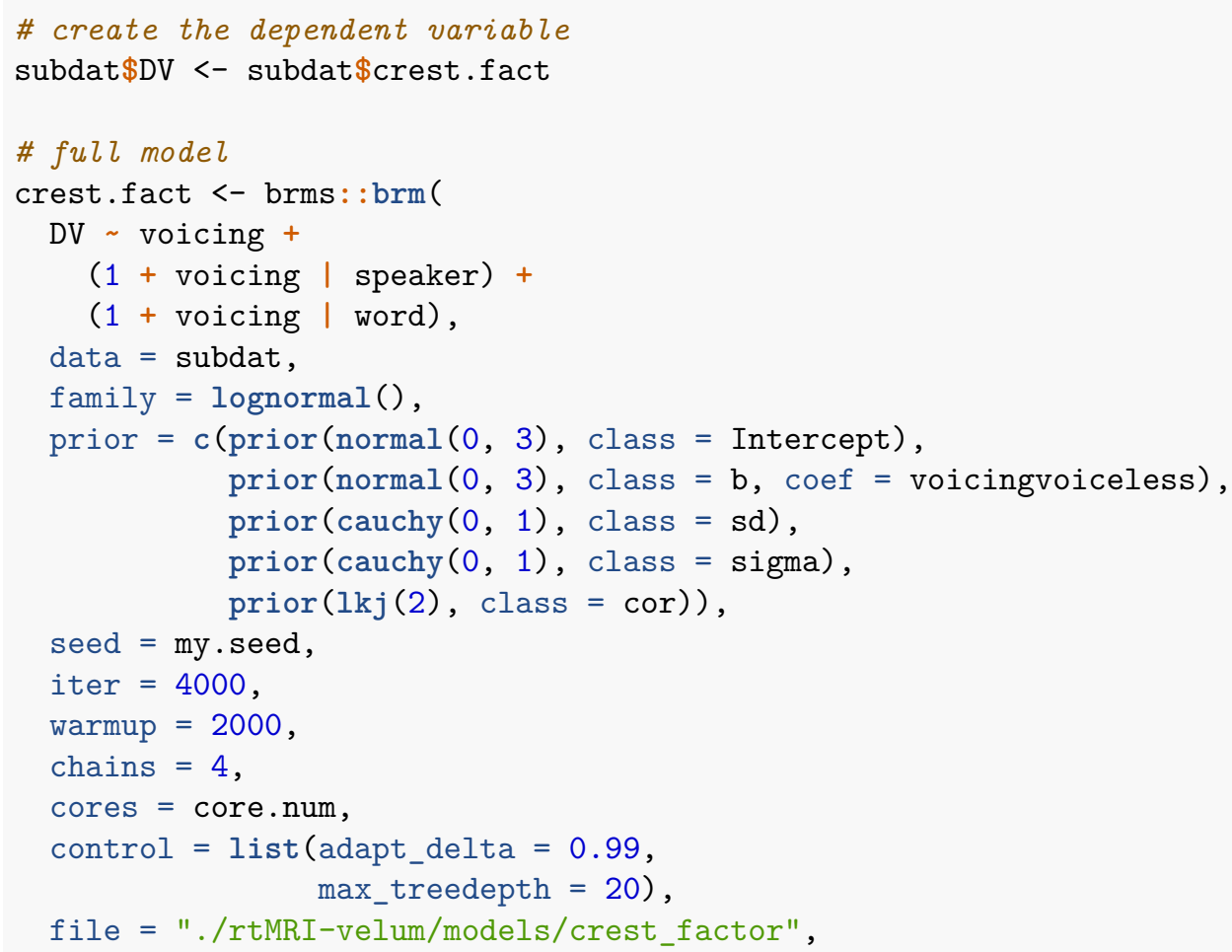




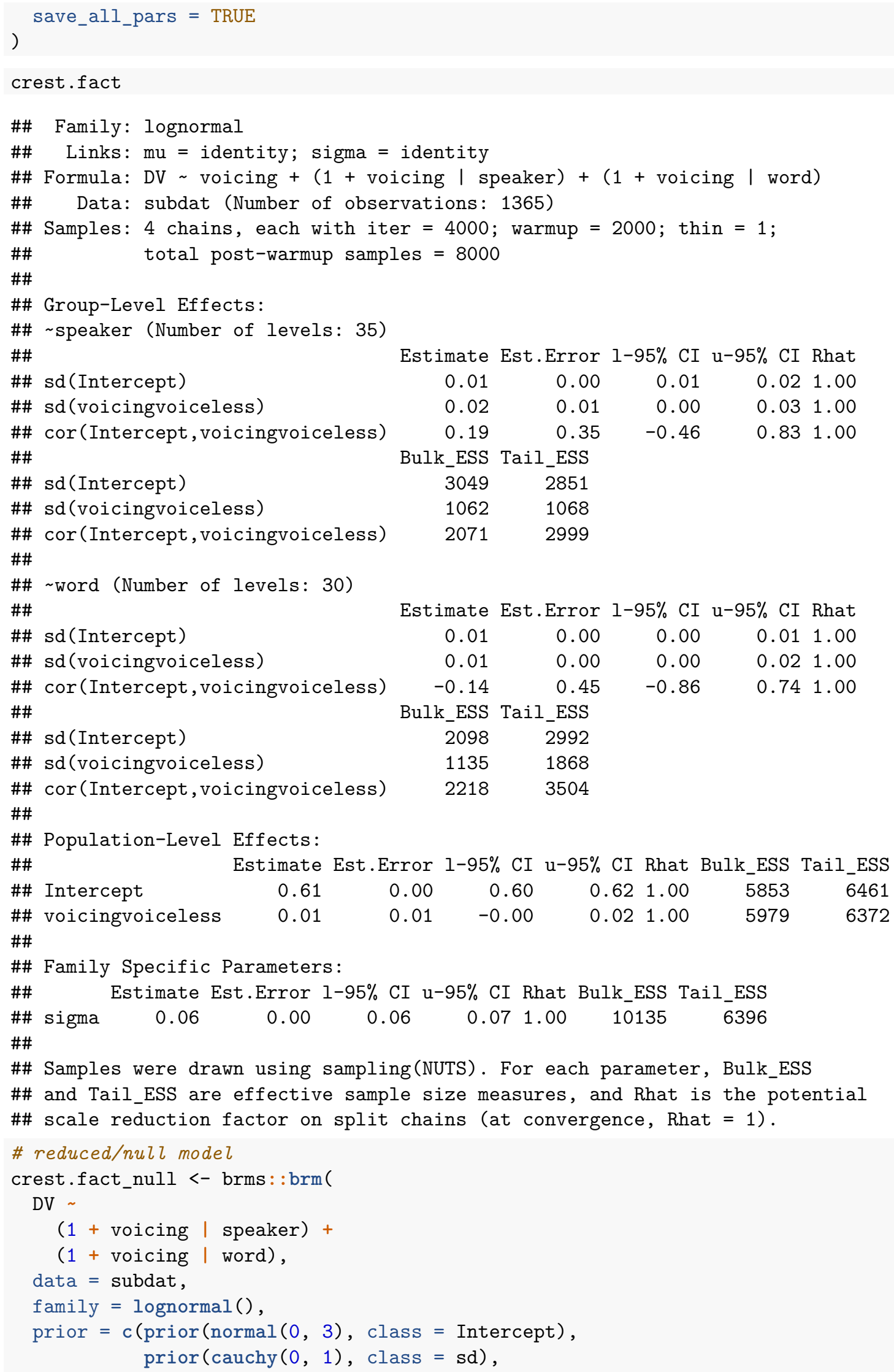




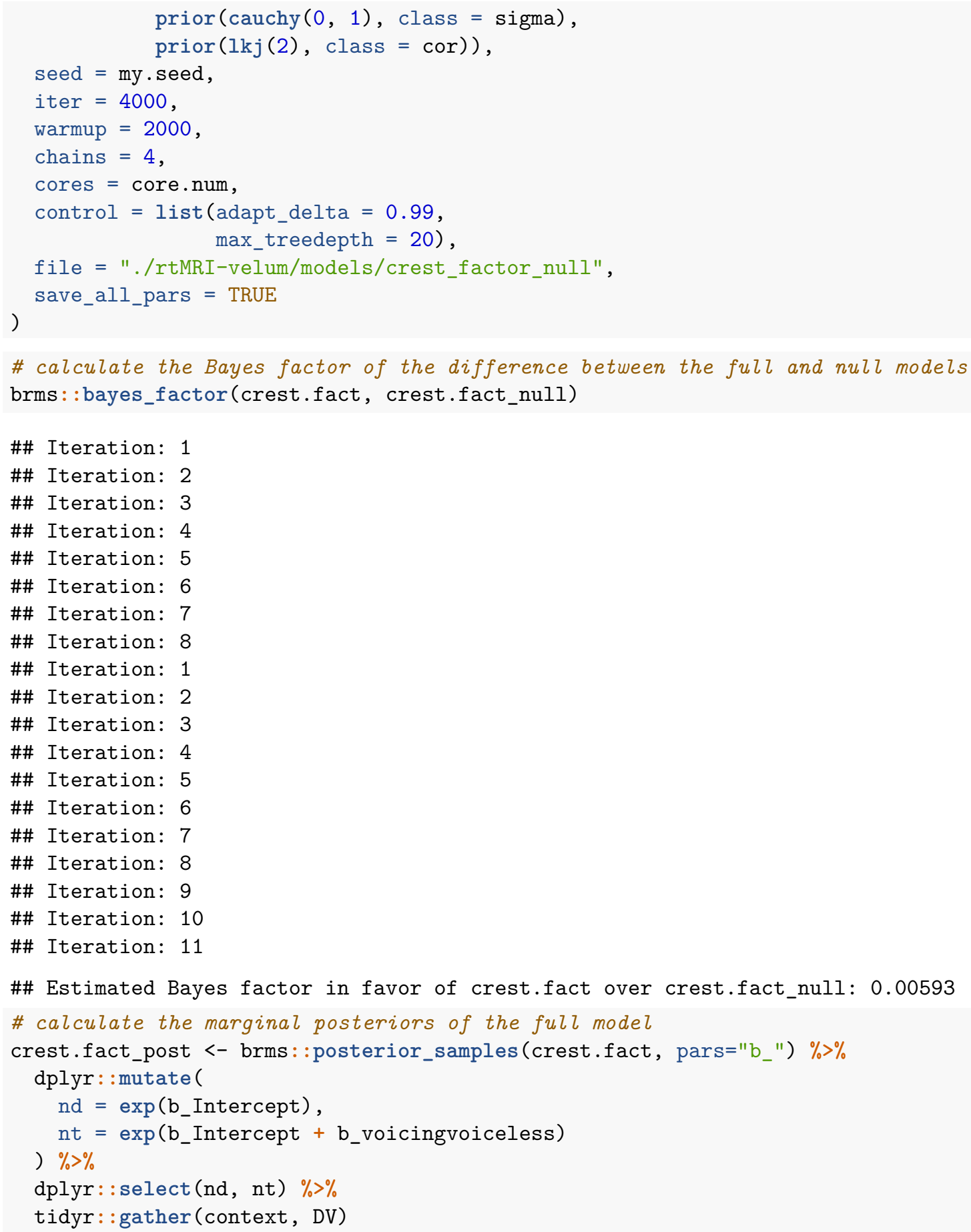

\subsection{Checks}

pp_check (crest.fact, nsamples $=50)+$ theme_minimal $($ ) 


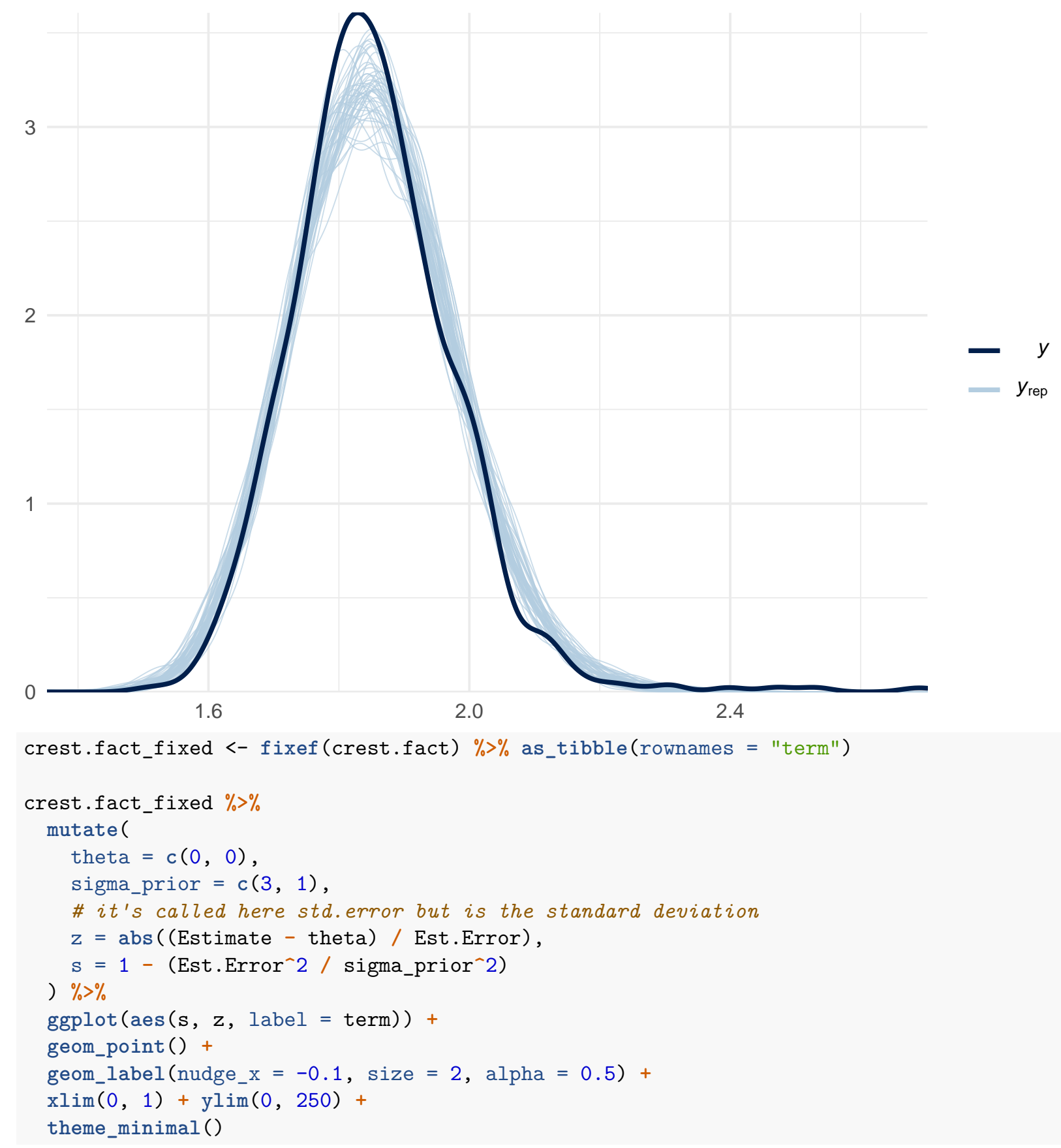




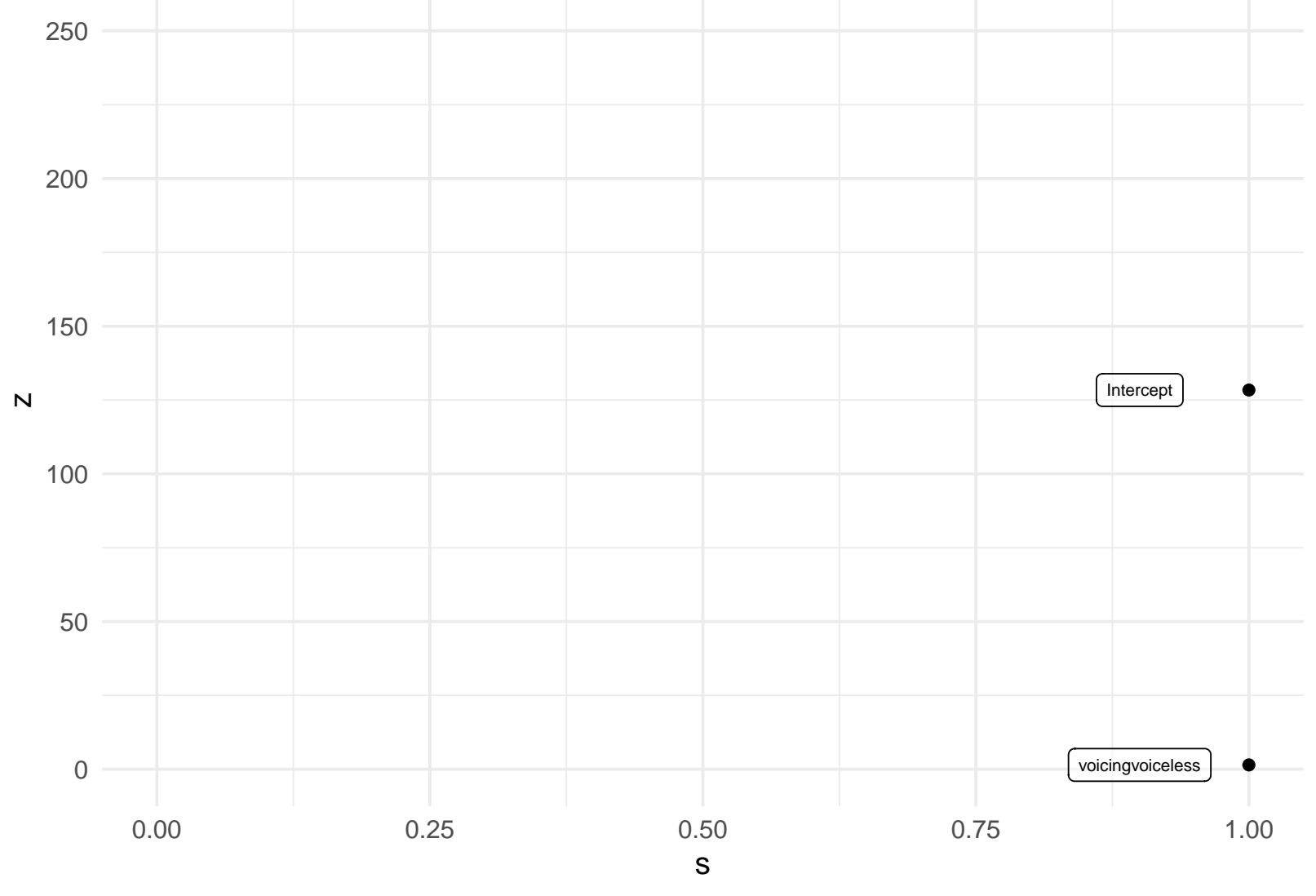

\section{Integral of velum movement in vowel (area under the curve)}

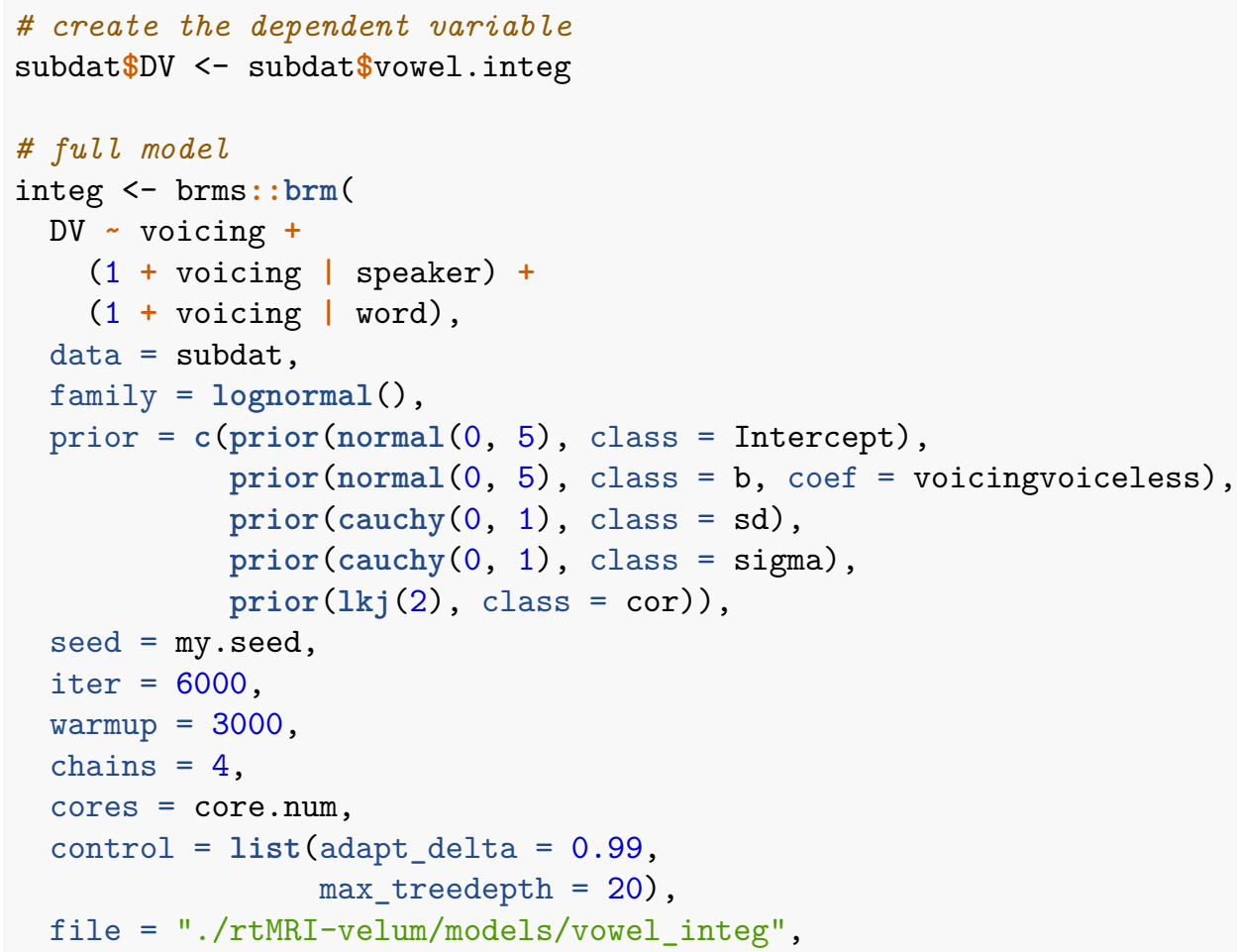




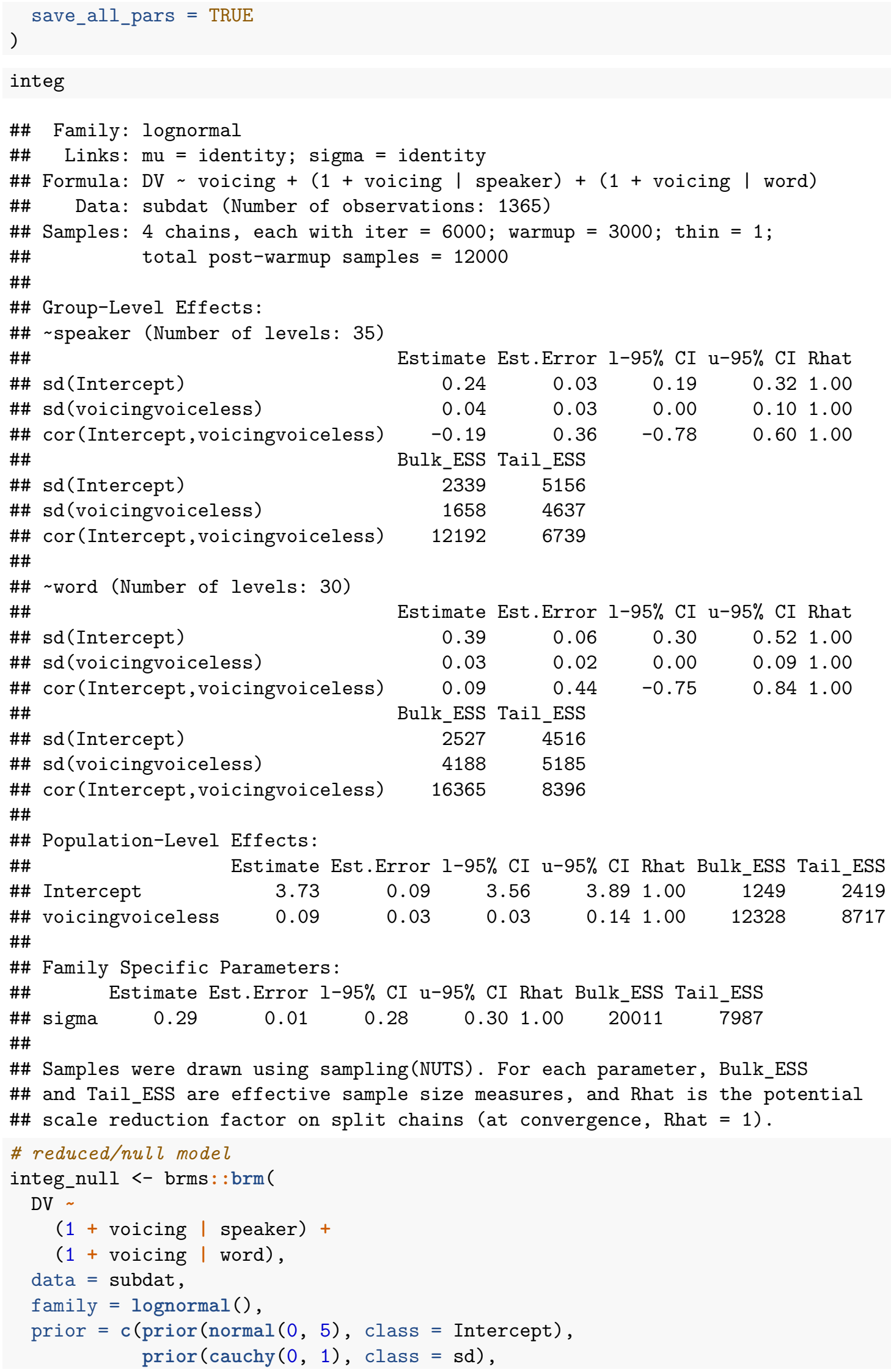




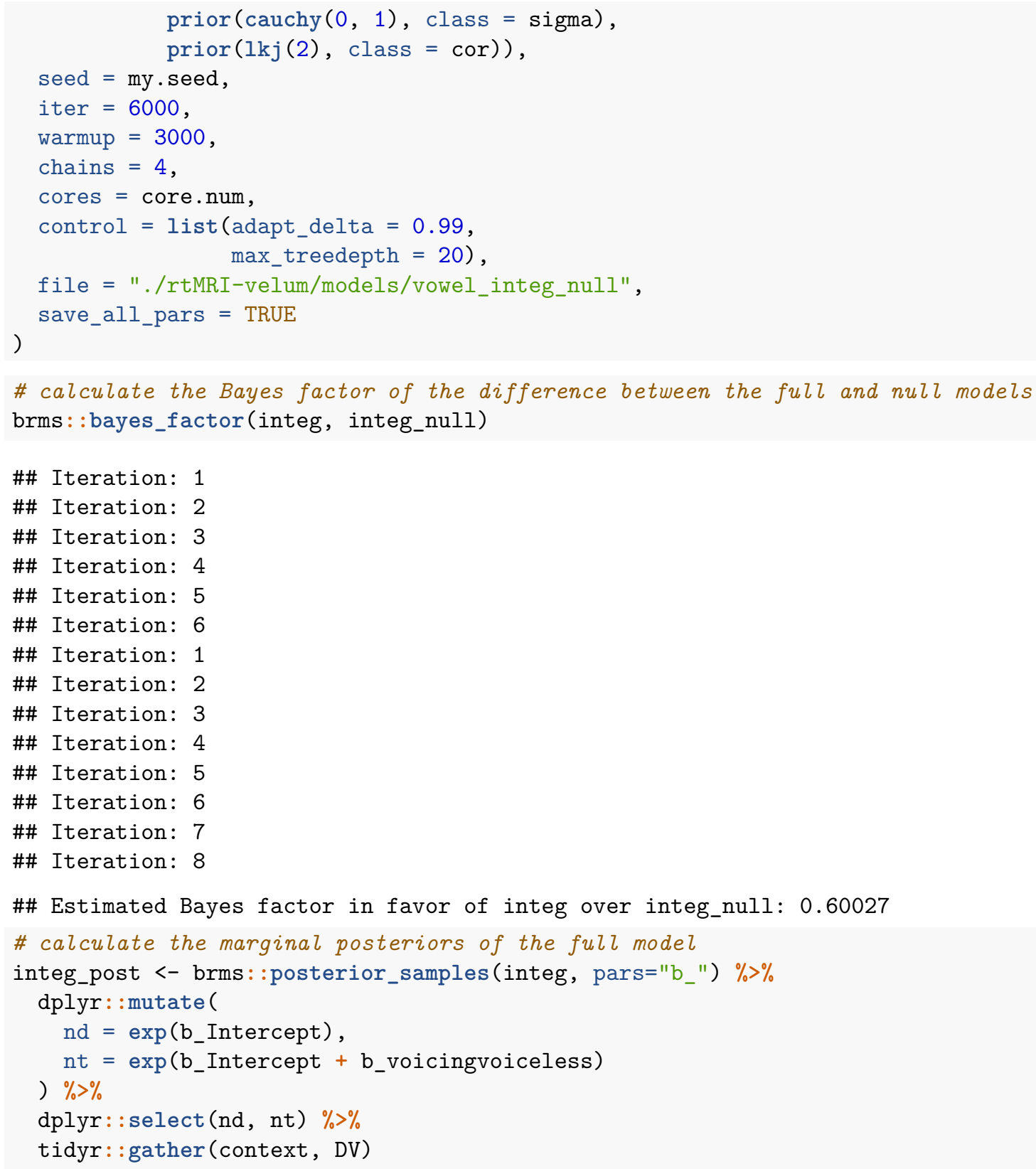

\subsection{Checks}

pp_check(integ, nsamples $=50)+$ theme_minimal $($ ) 


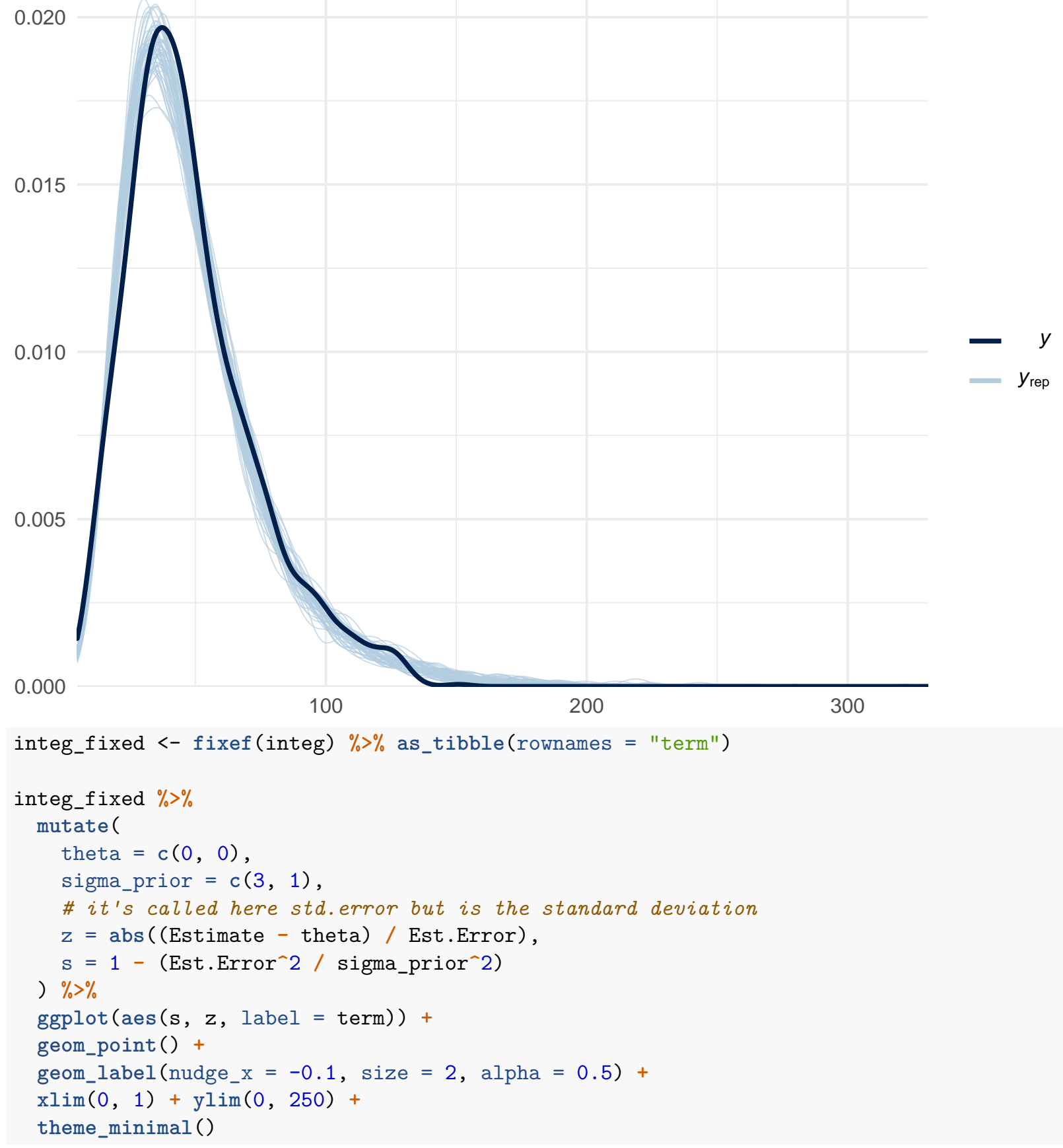




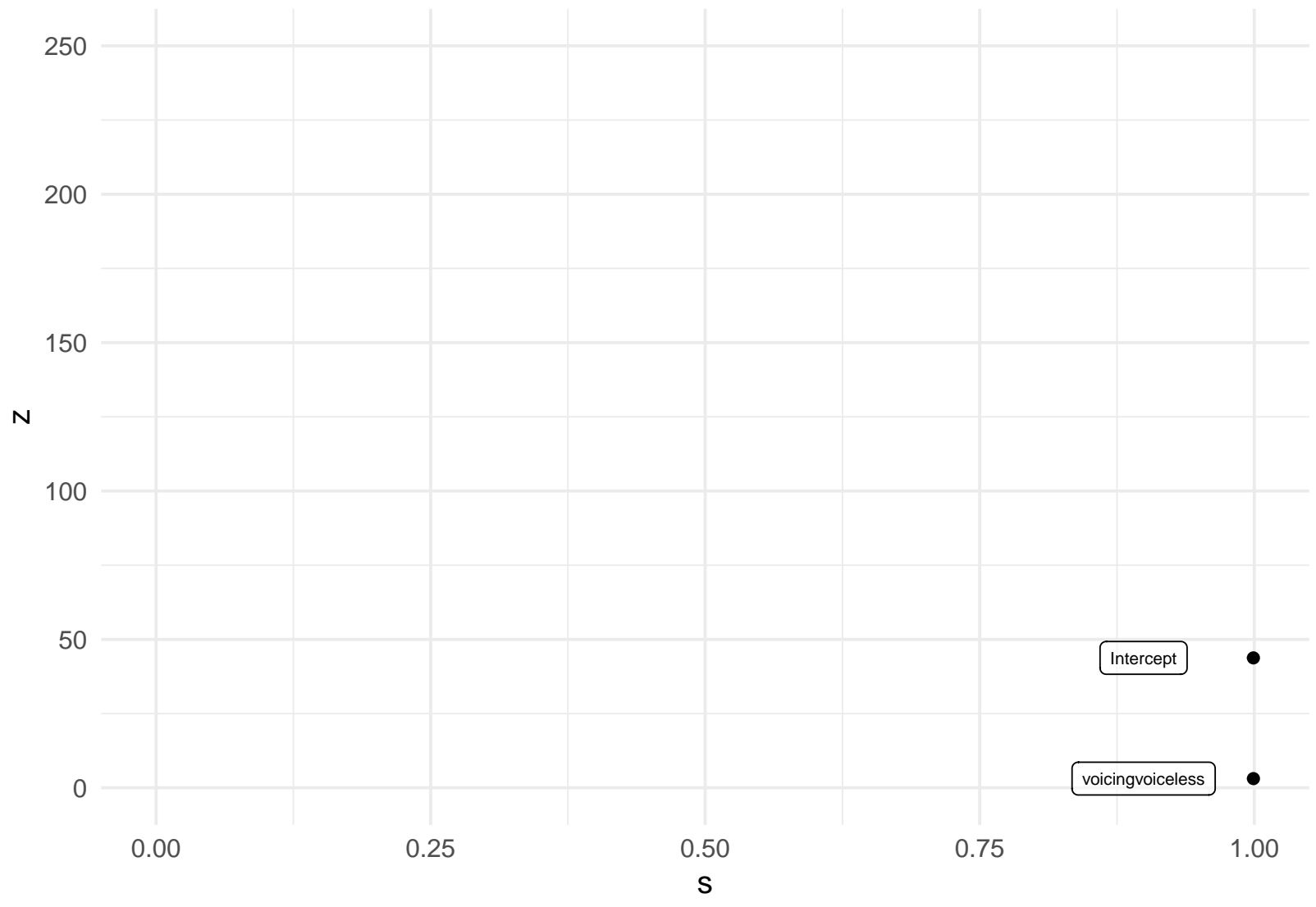

\section{Plotting posteriors}

\# colors to be used for plotting (suitable for B/W printing)

my.cols <- c("\#2c7fb8", "\#7fcdbb")

\subsection{Figure 5: duration, onset, peak (timing), offset}

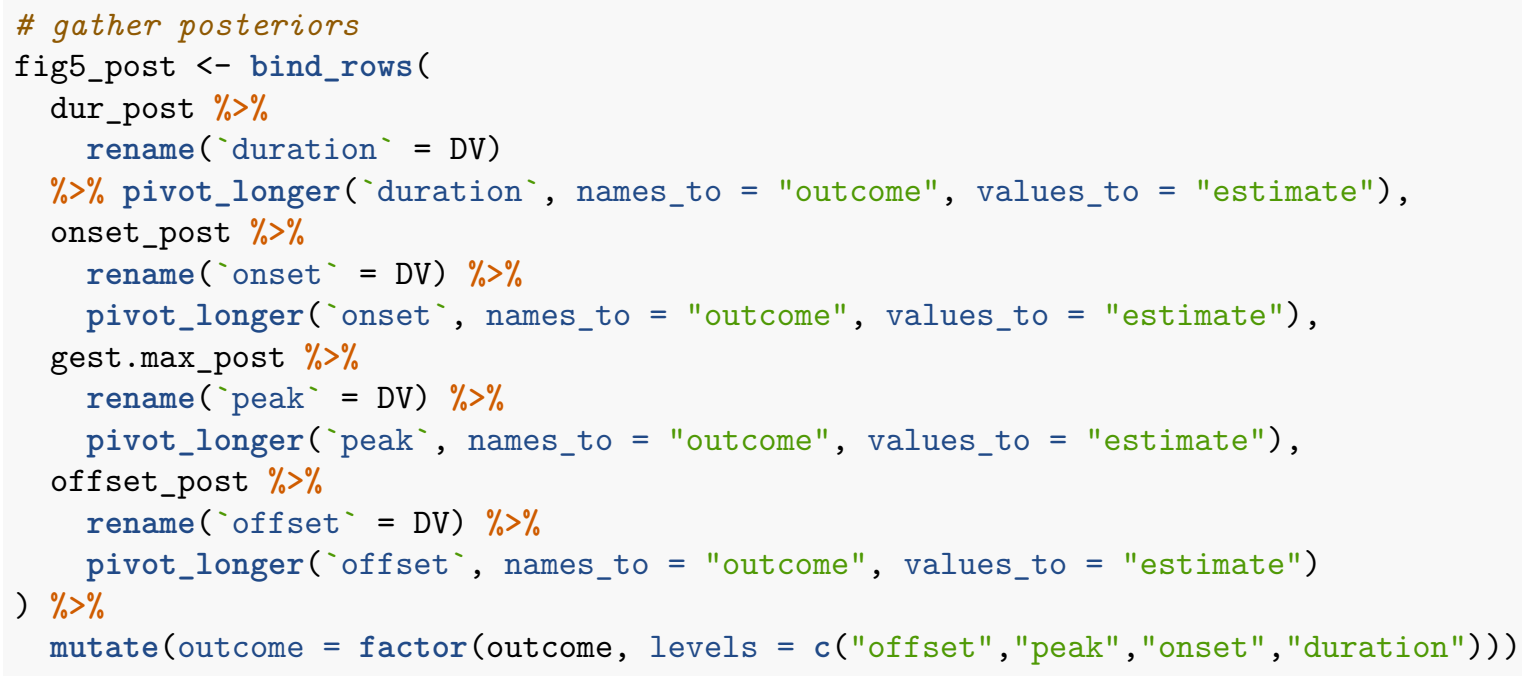




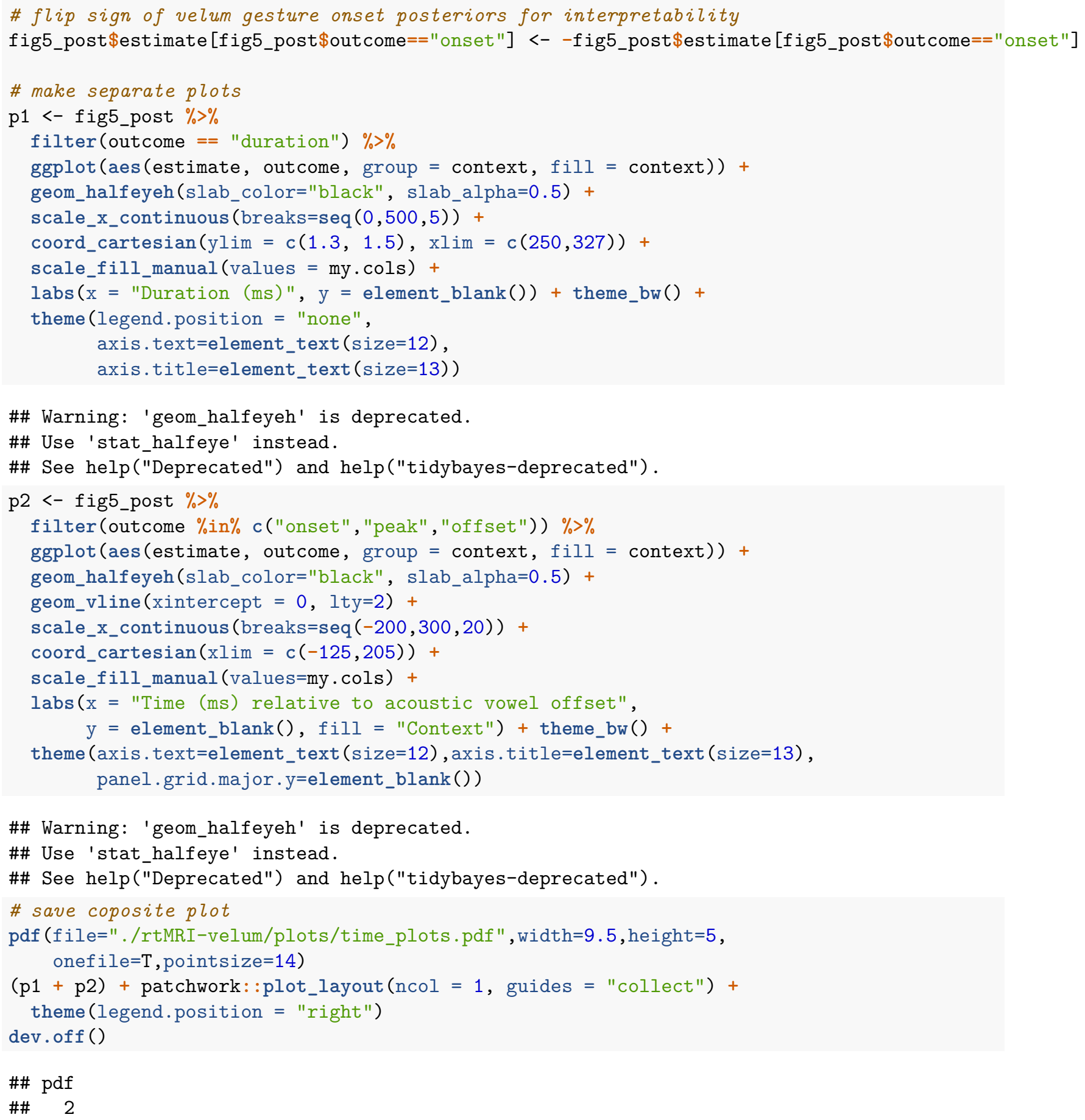

\subsection{Figure 6: peak (magnitude)}

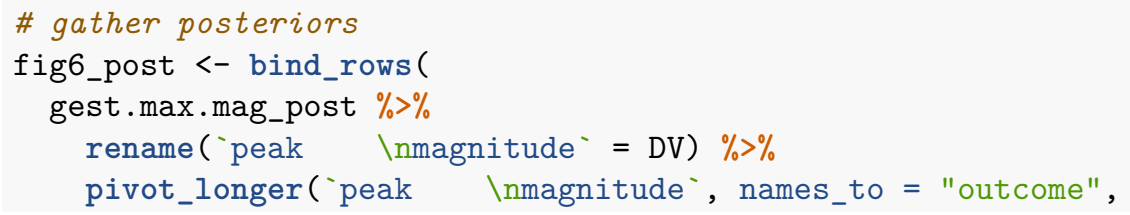




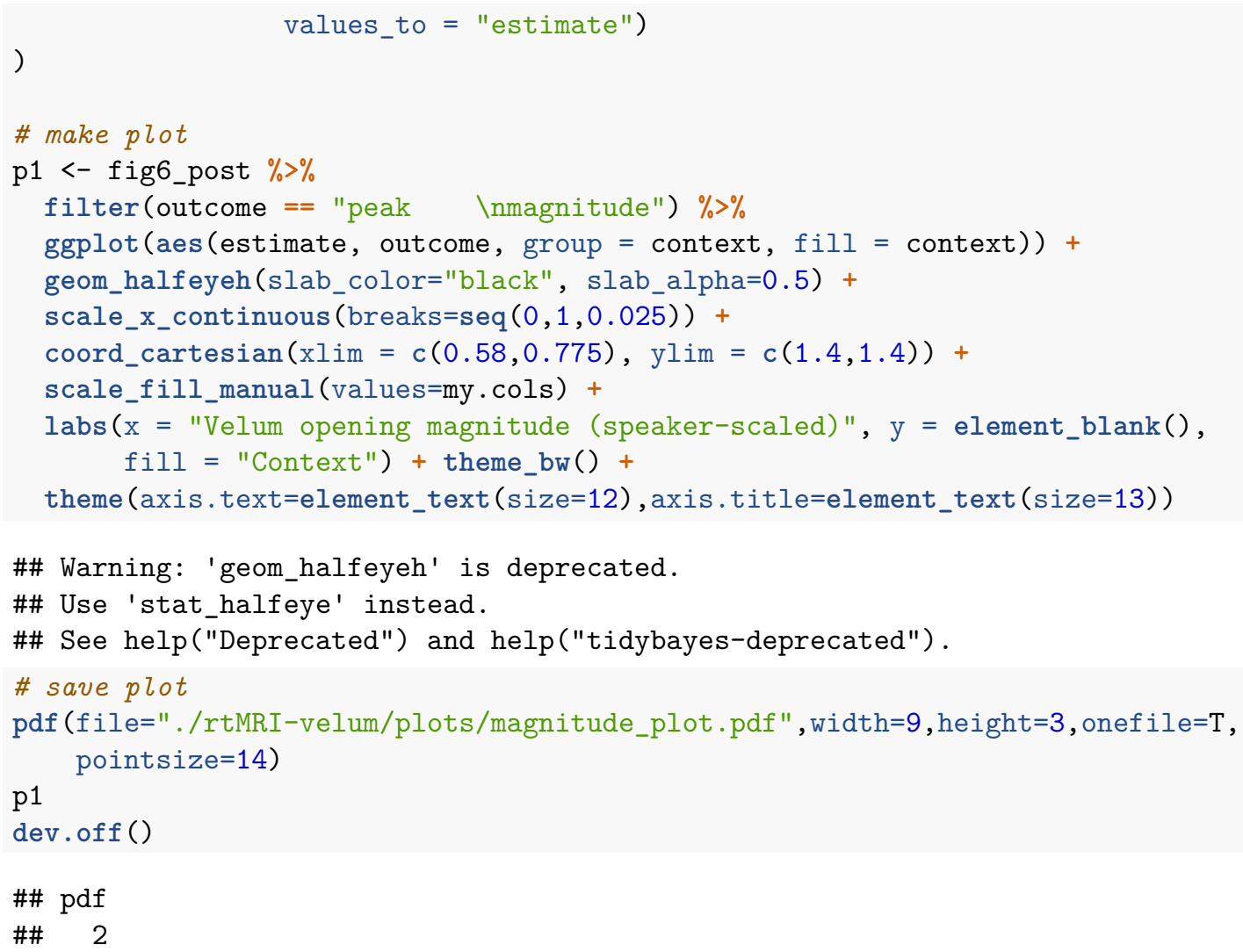

\subsection{Figure 7: opening stiffness, closing stiffness}

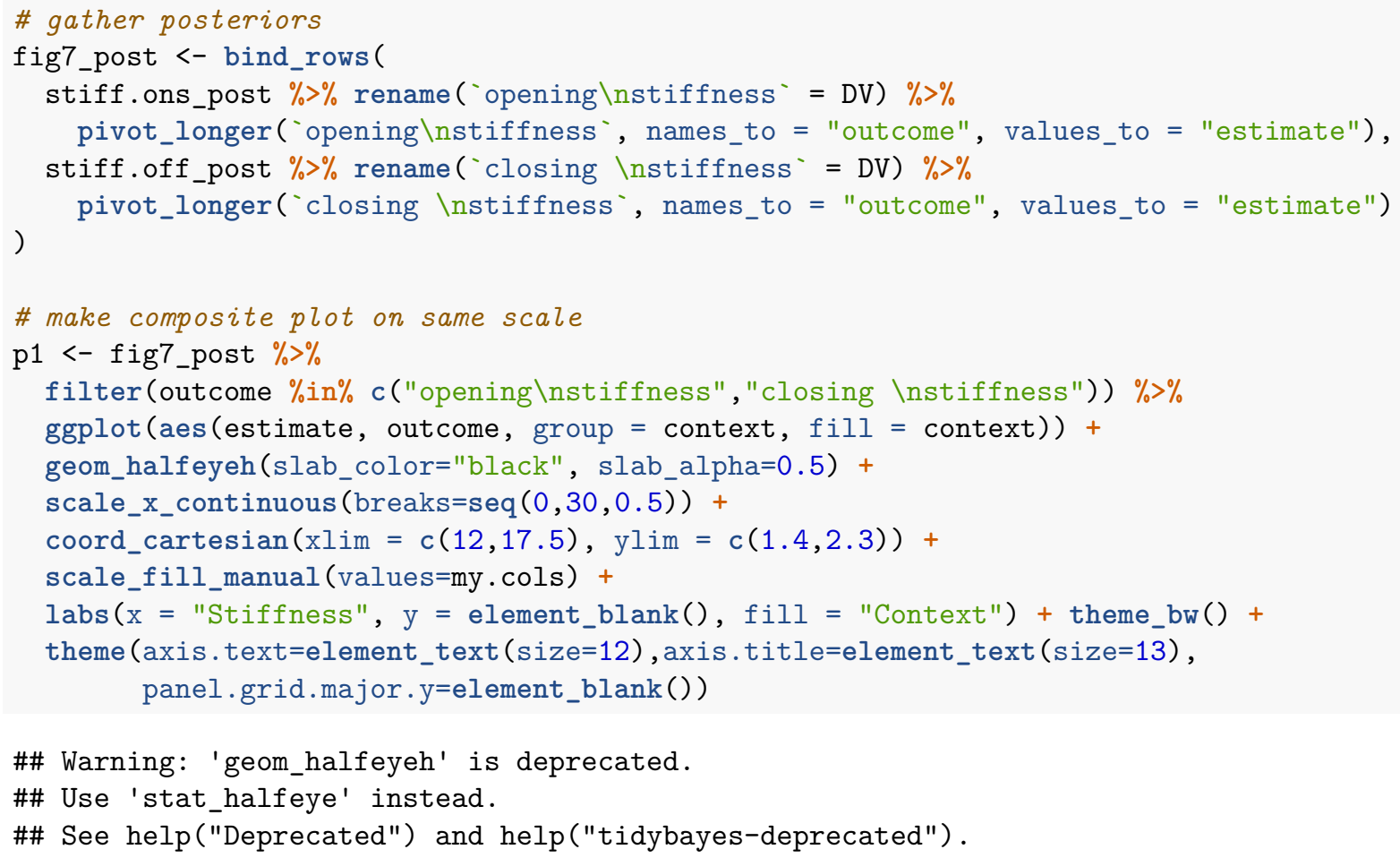




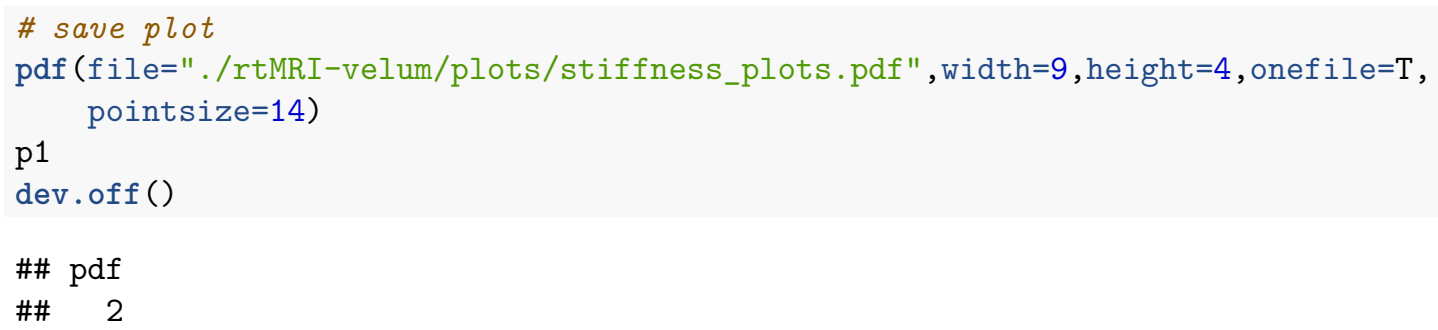

\subsection{Figure 9: kurtosis, crest factor}

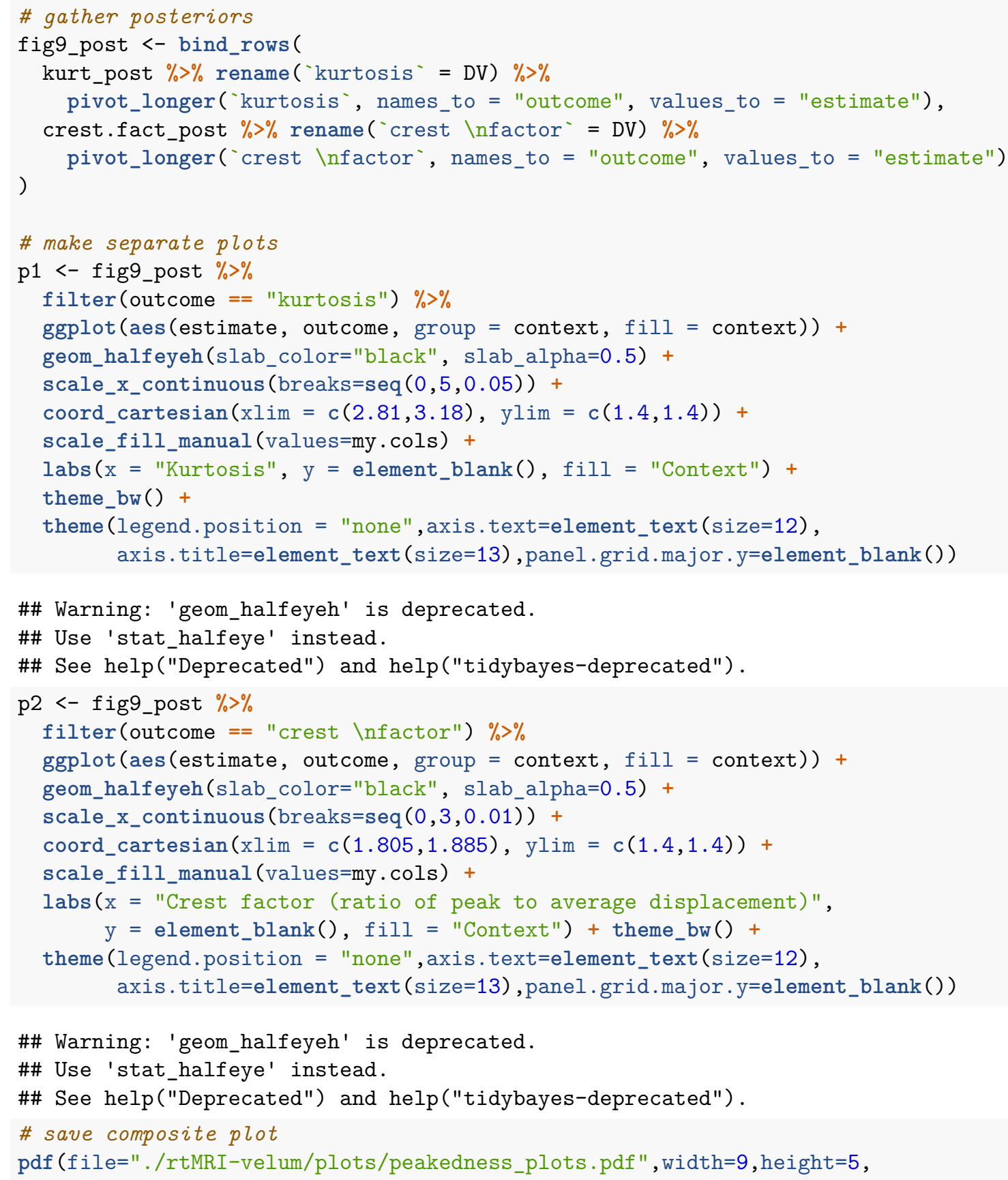




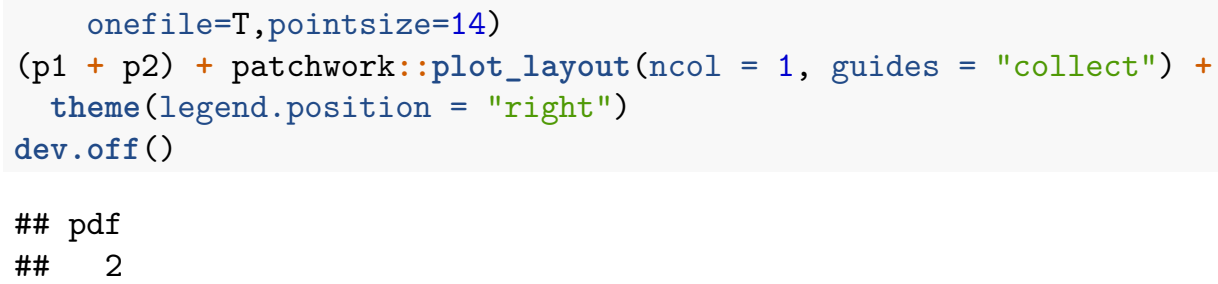

\subsection{Figure 10: velum displacement integral}

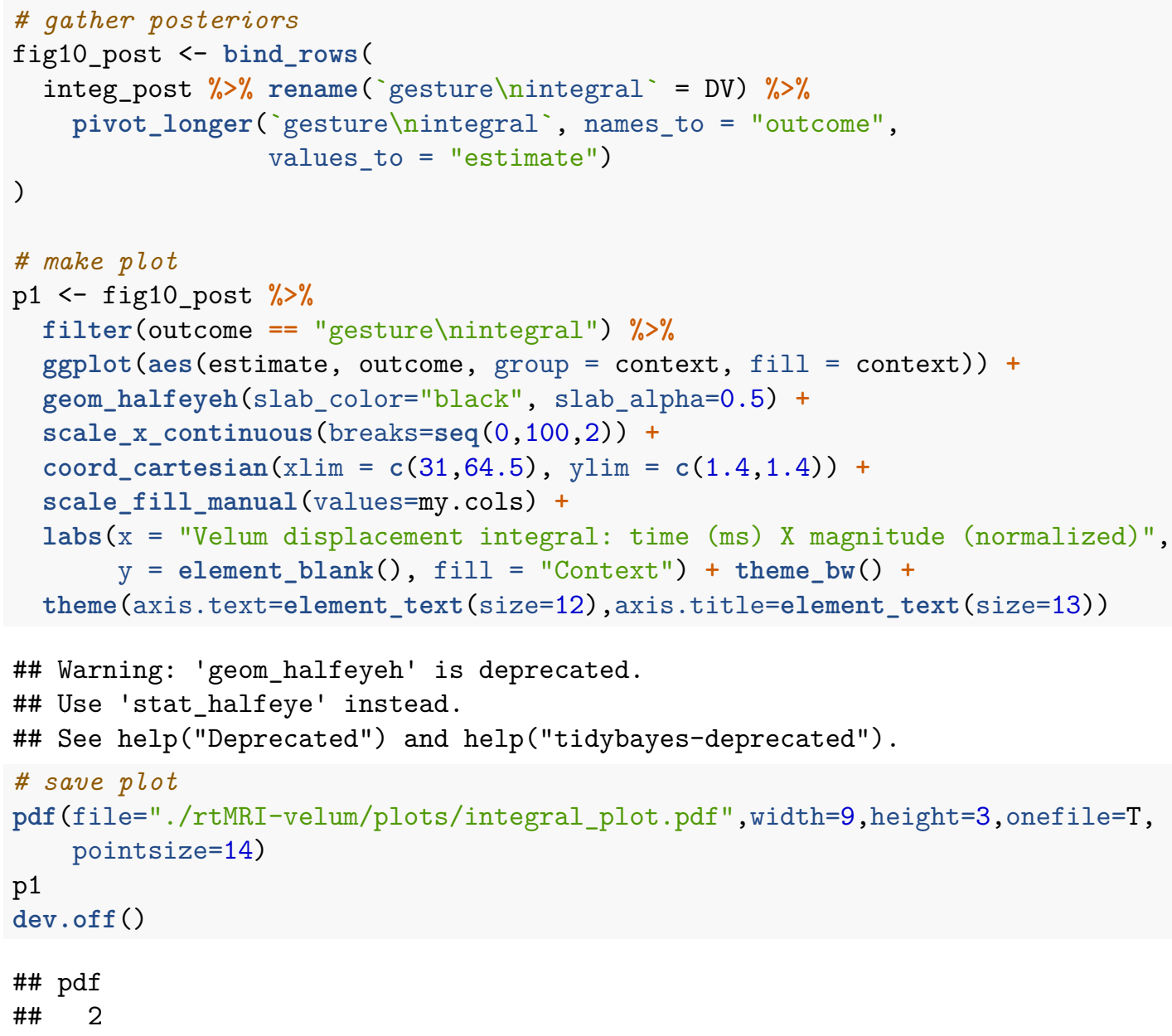

\section{References}

Betancourt, Michael. 2018. "Calibrating Model-Based Inferences and Decisions." arXiv preprint arXiv:1803.08393.

Gahl, Susanne, and R. Harald Baayen. 2019. "Twenty-Eight Years of Vowels: Tracking Phonetic Variation Through Young to Middle Age Adulthood." Journal of Phonetics 74: 42-54.

Ratnikova, E. I. 2017. "Towards a Log-Normal Model of Phonation Units Lengths Distribution in the Oral Utterances." International Research Journal 3 (57): 46-49. 
Rosen, Kristin M. 2005. "Analysis of Speech Segment Duration with the Lognormal Distribution: A Basis for Unification and Comparison." Journal of Phonetics 33 (4): 411-26.

Vasishth, Shravan, M. Beckman, B. Nicenboim, Fangfang Li, and Eun Jong Kong. 2018. "Bayesian Data Analysis in the Phonetic Sciences: A Tutorial Introduction." Journal of Phonetics 71: 147-61. 